\title{
The psychophysical inquiry into binocular summation*
}

\author{
RANDOLPH BLAKE $\dagger$ and ROBERT FOX \\ Vanderbilt University, Nashville, Tennessee 37240
}

\begin{abstract}
Experiments that compare monocular and binocular visual performance of human psychophysical Os on a variety of visual tasks are reviewed. The review attempts to include all experiments published in $\mathrm{E}$ nglish in this century, excluding work on stereopsis, rivalry, and evoked potentials. The concept of probability summation as a baseline for assessing the presence of neural summation is discussed, and the assumptions of several models for estimating probability summation are considered. Experiments are classified in terms of visual task, major categories being increment detection, flicker fusion, brightness magnitude, and contour resolution. A major conclusion is that binocular performance is superior for essentially all task categories and in most cases by a magnitude greater than that predicted by appropriate probability summation models.
\end{abstract}

The cardinal feature of the binocular visual system in man is the unification of the two separate monocular views into a binocular cyclopean view that betrays little trace of its monocular origins. Relatively little is known about the mechanism responsible for unification, although an examination of the physiology of the visual system reveals a number of features that seem expressly designed to promote unification. Some of the more prominent features are the partial crossing of the optic tracts at the optic chiasma and the elaborate system for the maintenance of conjugate eye movements. The existence of these features in man, and in binocular vertebrates in general, suggests an adaptive function of binocularity of sufficient magnitude to justify the survival of this complicated physiological organization. Yet the immediate advantage of having two eyes functioning as one is not obvious. The simple experiment of closing one eye does not result in any dramatic change in the appearance of the visual world. Rather, only by more stringent experimentation are the subtle advantages of binocularly joined eyes revealed.

The purpose of this paper is to review those experiments undertaken in the 20th century that have compared monocular and binocular performance on a variety of visual tasks. The rubric under which such experiments are typically grouped is "binocular summation." These experiments compare the relative performance on some visual task (such as detection or form resolution) by a human $S$ using one and both eyes. In the literature, this kind of inquiry into binocular vision is treated separately from investigations of stereopsis and of binocular rivalry; following that distinction, the voluminous research on these binocular phenomena is not included. A further exclusion is data

*This work was supported by a NIMH predoctoral fellowship to Randolph Blake and by Public Health Service Grant EY00590 to Robert Fox. We would like to thank Joseph S. Lappin for his helpful comments.

$\div$ Now at the Department of Ophthalmology, Baylor College of Medicine, Houston, Texas 77025 .

$\div$ Request reprints from Robert Fox. Department of Psychology. Vanderbilt University. Nashville, Tennessee 37240. on binocular interaction assessed by evoked cortical potentials. This growing research area, while of general relevance, involves special issues that are best treated separately.

Finally, there is the inevitable gray area containing papers that resist convenient classification and thus pose questions about their warrant for inclusion. In some instances, relevant data may be embedded in some experiments and quite difficult to extract, because the experiments were directed to an issue other than binocular summation. Or the experimental conditions were joined in unusual ways (e.g., the eyes were in different states of adaptation) that preclude comparison with other work. Given these constraints, the policy has been to assume a liberal criterion and to include papers whose relevance may be marginal. Even so, the necessarily indistinct definition of relevance makes it likely that some papers have been overlooked. ${ }^{1}$

It should be noted that the review attempts to encompass articles that have appeared in English-language journals in this century. A review of older studies, especially those in German, has been made by Roelofs and Zeeman (1914).

This paper is organized in the following way: First, a theoretical context is developed for considering these experiments and some working definitions are developed. Second, the experiments are divided and categorized in terms of the visual task employed to measure summation, and wherever possible an effort is made to reach some conclusions. Third, a final section offers some general conclusions about the status of the work in this area and offers some suggestions for further inquiry.

\section{AN IMPLICIT ASSUMPTION UNDERLYING BINOCULAR SUMMATION EXPERIMENTS}

The majority of experiments on binocular summation were not guided by an explicit, highly developed theoretical framework from which specific predictions were derived and tested. Instead. an empirical interest in 
the quite reasonable question, "Are two eyes better than one?" has served as a sufficient raison d'etre for experimental attack.

Yet simply raising that question implies a theoretical context. The context emerges from two considerations that suggest that binocular performance should be superior to monocular performance. First, the anatomical connections between eye and cortex provide for the convergence of the monocular inputs from corresponding portions of each retina upon a common cortical target. Second. physiological data concerning neural interaction at a point of convergence within a system (e.g., that of the spinal cord reflexes) provides strong evidence that anatomical convergence supplies the substrate for functional neural interaction. Following from both the anatomical and physiological prerequisites for interaction, it is reasonable to assume that behavioral experiments should provide evidence for the underlying neural interaction. This general assumption undoubtedly influenced many of the specific experiments on binocular summation, even though the assumption may not have been explicit in the published reports. The application of the term "binocular summation" to these experiments probably reflects the pervasiveness of this assumption. More specific evidence can be obtained from a contextual analysis of particular experiments where the results did not reveal summation or where the amount of summative performance obtained was less than anticipated. Often these discussions consider potential procedural inadequacies to explain the failure to obtain the anticipated summation and do not entertain the possibility that the hypothesized neural interaction may be unrelated to the function of the system as it is assessed behaviorally.

Even though research on binocular summation has not been closely linked to explicit theory, it would be useful to impose a heuristic theoretical context on binocular summation experiments in order to talk about them in a consistent way. The existence of implicit theoretical assumptions concerning summation suggests that neurophysiological concepts of neural interaction would provide an appropriate context.

In an earlier draft of this paper, we attempted to provide a context by linking various magnitudes of binocular performance with a number of concepts derived directly from the study of neural interactions, particularly those found in spinal reflexes. These concepts are facilitation, linear or additive summation, occlusion, and inhibition. They all characterize the events that may occur when two afferent inputs converge upon a common set of neural units, the response of the system depending upon the total activity generated in the common neural pool. Facilitation refers to the case where the output of the common pool is greater than the sum of the afferent inputs. Linear summation refers to the case where the output of the common pool is the sum of the two afferent inputs. Occlusion refers to the case where the output of the common pool is less than the sum of the afferent inputs yet greater than the response produced by a single input. Inhibition refers to the case where the output of the common pool is equal to or less than the response generated by a single input.

It is tempting to draw a parallel between the neural operations that give rise to these concepts and the binocular visual system. Monocular pathways can be thought of as the afferent inputs, and the common cortical neurons upon which the pathways impinge can be thought of as the common neuronal pool. Yet, one difficulty with this kind of comparison is the enduring problem of relating behavioral data to underlying neural mechanisms. Rather strong and largely untestable assumptions must be made in order to talk about the data of binocular summation experiments in terms of the well-defined neural concepts of facilitation, occlusion, and so on. Nevertheless, these concepts may be valuable in characterizing varying magnitudes of binocular performance. While this usage would not involve explicit physiological assumptions, it does introduce a second difficulty. This concerns the properties of the measurement scale used for assessing binocular performance. Binocular tasks involving threshold measurement typically use indicators such as the probability of correct performance, while suprathreshold binocular tasks such as brightness matching use indicators defined on some arbitrary scale such as luminance units. It is not at all clear how these different kinds of indicators could be reconciled or combined to yield a common fundamental scale of binocular performance. This issue, of course, is part of the more general problem of providing appropriate measurement models for psychological attributes. It is our impression that the data on binocular summation are not in a form that lends itself to serious examination within the context of measurement theory, and for that reason a serious attempt at characterizing the magnitudes of binocular performance in terms of the neural concepts or labels would be premature.

These considerations discouraged any sustained effort to impose a general theoretical context on binocular summation experiments. This does not mean, of course, that specific models for specific kinds of binocular tasks should not or could not be developed.

At present, the main theoretical issue concerns the interpretation of binocular performance as indicative of neural interaction mediating the confluence of the monocular inputs. Since binocular performance may be superior to monocular on probabilistic grounds that do not require neural interaction (a point considered in detail in the next section), demonstration of facilitatory neural interaction requires the magnitudes of binocular performance to be significantly greater than anticipated by probability. The term "neural summation" is generally used to refer to such interaction.

\section{Probability Summation}

Interpretation of superior binocular performance in 
terms of probability is now generally referred to as the probability-summation model. The basic concept is that some improvement in binocular performance relative to monocular is to be expected, because the simultaneous presentation of inputs to both eyes provides the $O$ with two opportunities to make a detection or otherwise produce a correct response. The additional opportunity will tend to yield superior binocular performance. Because the monocular stimuli are presented simultaneously under the binocular condition within the time-and-rule structure of a single trial, it may not at first be obvious that the $O$ has, in fact, two chances to perform correctly on that trial. But the probability-summation model asserts that the binocular stimulation condition is logically the same as though one eye received two successive stimulations and the two corresponding responses are combined by a rule that says that successful performance on either the first presentation or the second presentation or on both presentations will be counted as one successful response.

Although some concern about the operation of probability was expressed early in the research on binocular summation (e.g., Dawson, 1913), Pirenne (1943) has received credit for the first explicit formulation of the probability-summation hypothesis. He pointed out that, from the classical independence theorem of probability theory (e.g., Feller, 1960), a substantial improvement in binocular performance would be the expected result. The independence theorem says that the expected value of two independent events is equal to the union of these events minus their intersection. Thus, if the left-eye detection probability is .50 and the right-eye detection probability is .50 , the combination of these monocular probabilities leads to an expected binocular detection probability of .75 ; that is, the sum of the left-eye and right-eye probabilities minus their product yields the expected binocular detection probability of .75 .

The logic underlying the probability-summation hypothesis cannot be denied, and recognition of it as an alternative to neural summation is generally acknowledged in the contemporary work on binocular summation. The general practice has been to use the expected binocular performance computed from probability considerations as a baseline for evaluating empirical performance. If the performance exceeds that predicted by probability summation, the data are interpreted as evidence for some form of neural summation. Thus, the probability-summation model has served to form a normative baseline against which neural summation effects must be evaluated.

Given such a normative baseline, it is proper to inquire as to whether the norm is appropriate for the empirical conditions of the binocular summation experiment. In recent work, questions have been raised about the applicability of the classical probability theorem to experiments involving measurement of thresholds. A major criticism of the application of the theorem, raised by Eriksen (1966), is that it tends to overpredict the level of performance expected from two independent chances to perceive by giving double weight to the guessing factor. That is, the probability of a correct response on a single observation trial (monocular trial, for vision) is composed of at least two parts: (a) trials on which the $O$ correctly perceived and (b) trials on which the $O$ did not perceive but did guess correctly. These two parts make up each monocular probability. When the two monocular probabilities are then combined by the theorem to predict the binocular probability, the guessing component is included in each. But, during actual binocular viewing, the $O$ would guess only if he failed to perceive with both the left and right eyes. Thus, the binocular performance predicted from the classical theorem is inappropriate, Eriksen argues, for psychophysical tasks involving a guessing component, since the theorem inflates the baseline for independence.

By applying the standard correction for guessing to the classical theorem, it is simple to derive an independence model that is applicable to psychophysical tasks, the resulting model being the two-state high-threshold or decision-threshold model (Eriksen, 1966; Green \& Swets, 1966). On this model, it is assumed that on any single trial the $O$ is in one of two states, (a) the correct-perception state with associated hit rate (HR) of 1 , or (b) the guessing state with HR equal to the a priori probability of a correct guess. The probability, $\mathrm{P}(1)$, of a correct response, then, on a single trial is

$$
P(1)=C+1 / n(1-C),
$$

where $C$ is the probability of being in the correct-perception state after the single trial and $1 / \mathrm{n}$ is the probability of a correct guess. Next, consider the situation where the $O$ is given two chances to perceive before reaching a decision. Assuming that the two observations are independent, it follows that the predicted probability, $\mathbf{P ( 2 )}$, of a correct response following the two observations is

$$
P(2)=2 C(1-C)+C^{2}+(1-C)^{2} 1 / n,
$$

where $2 \mathrm{C}(1-\mathrm{C})$ is the probability of being in the correct-perception state following either the first or the second observation, $\mathrm{C}^{2}$ is the probability of being in the correct-perception state following both the first and the second observation, and $1 / n(1-C)^{2}$ is the probability of being in the correct-perception state on neither observation, yet guessing correctly.

Unlike the classical probability theorem, this expression is logically consistent with the conditions of threshold experiments in that the guessing component is properly represented only once. This fact, in turn, raises the possibility of employing the two-state model as a normative baseline to test for binocular summation in excess of probability. (For the binocular situation. the 
correct form of this high-threshold, two-state model is given in Appendix A.)

Note that the model assumes the $\mathrm{O}$ to be in either a correct-perception state or a guessing state. But, contrary to that assumption, considerable contemporary theoretical experimental work strongly suggests that sensitivity varies continuously or at least is represented by many states. A probability summation model designed expressly to accommodate multiple states has been described by Eriksen (1966). This model, referred to here as the multistate model, takes the following general form. The initial assumption is that Os can distinguish several different subjective states of confidence about their judgments of stimulus presentation. Differences in confidence, which can be determined by rating states of confidence, seem to reflect different states of perceptual sensitivity in that the accuracy of performance covaries with the confidence rating of the $O$. The best performance (indexed by some measure such as percent correct detections) is associated with the most confident state: as performance declines, confidence decreases. One implication of multiple states of perceptual sensitivity varying over trials is that a probability model that assumes only two states, a correct detection state and a guessing state, will tend to overpredict the performance level based upon two opportunities to perceive. The multistate model deals explicitly with the presence of multiple perceptual states by computing the joint occurrence of the various perceptual states of each eye and expressing the results as a linear combination of the proportion of times such a particular state occurs weighted with the performance measure associated with each of the states. Granting the assumptions that the perceptual states of each eye are independent and that the $O$ 's report will be based upon the most sensitive of two independent perceptual states, an increase in performance associated with two independent chances to perceive is due to the increased likelihood of entering a more sensitive perceptual state on one or more of the two chances. This form of the multistate model has been tested in visual perceptual tasks where the two inputs are forced to be independent by virtue of their temporal or spatial separation, and close agreement has been found between the obtained increase in performance and that predicted by the model (Eriksen, 1966; Eriksen \& Lappin, 1965, 1967).

The formulation of the multistate model has been strongly influenced by the general implications of the theory of signal detectability (e.g., Green \& Swets, 1966; Swets, 1964). Indeed, within the framework of signal detectability theory, a probability summation model can be found known as the integration model. This model specifies an $O$ 's detection performance in situations where more than one observation of the signal is permitted. Specifically, the model predicts the overall sensitivity $\left(d^{\prime}\right)$ resulting from $n$ independent observations by integrating the $n$ independent measures of sensitivity. For binocular vision, this entails combining the two monocular sensitivites ( $d^{\prime}$ monocular) to yield the expected binocular sensitivity (d' binocular). The relationship between $d^{\prime}$ monocular and $\mathrm{d}^{\prime}$ binocular is $\mathrm{d}_{\text {bin }}^{\prime}=\sqrt{\Sigma \mathrm{d}_{\text {mon }}^{\prime}}{ }^{2}$. Thus, the expected $d^{\prime}$ is based on the integration of information from the separate and independent monocular observations.

Both the integration model and the multistate model are similar in that neither assumes a theoretical dichotomous sensory threshold below which responses are governed by pure guessing. In both formulations, confidence ratings of an $O$ play an essential role in estimating variations in perceptual states.

Although the models share assumptions and have a common theoretical orientation to the threshold experiment, they are not strictly equivalent. For instance, the usual application of signal detection theory involves assumptions about underlying processes (e.g., noise and signal-plus-noise probability density functions), while such assumptions are not made by the multistate model. Further, on the integration model, the $O$ 's response is derived from the accrual of information from each successive observation, yet on the multistate model, the O's decision is based on the observation associated with the most sensitive perceptual state. Considering these differences, it is not surprising that the models yield somewhat different predicted values for statistical independence. And such differences have been found by Keeley and Doherty (1968) for a series of monocular form-recognition tasks using multiple observations.

Recently, Keeley and Doherty $(1968,1969,1971$ a, b, 1972) have developed a model similar in spirit to the multistate and integration models that seems applicable to binocular summation. Their model is based on the assumption that in multiple observation situations an individual makes an implicit response following each observation in that the responses are independent. Conditional probabilities of an implicit response, given a particular stimulus, are estimated from single observation hit rates and then combined by Bayes's rule to predict the hit rate following multiple observations. Although this Bayesian model has been applied only to multiple observation form-recognition tasks, there appears to be no reason why the model could not be extended to binocular summation situations. To our knowledge, however, this application has not yet been made.

Considering the appropriateness of the classic probability model (e.g., Pirenne, 1943) with the more contemporary multistate model proposed by Eriksen and the integration model of signal detection theory, it seems clear that the classic model does not apply and should not be used whenever "guessing" is a component in detection performance. Guessing is explicit in forced-choice methods, but it is also operative in situations where false alarms occur. On the theoretical 
analysis of the threshold experiment provided by the theory of signal detection in its several formulations, false alarms are an inevitable consequence of confusion between noise and signal. Accordingly, the classic model would not be appropriate in general. Since there is considerable support for the theory of signal detection analysis, it is difficult to specify a reasonable case where the classic model would apply. At the very least, it seems incumbent upon an advocate of the classic model to justify its application, especially when attractive alternatives are now available.

A concern about the appropriate model for estimating probability summation is not, of course, limited to the case of binocular vision. Thus, Guth (1971) has pointed out that questions about summation models would also apply to assessing the independence of chromatic detection mechanisms, and in response to Guth's note, Braddick (1972) has offered additional comments. Finally, the question of probabilistic interaction is by no means restricted to vision research, but arises whenever information is processed simultaneously. A thoughtful review of this general problem is provided by Gamer and Morton (1969).

\section{The Double-Energy Monocular Condition}

The probability summation models described in the previous section provide a baseline for determining whether an increase in binocular performance relative to monocular is sufficient to warrant the interpretation that neural summation is operative. In this section, we consider a second kind of baseline that has frequently been employed to estimate the magnitude of neural summation observed in particular experiments. This second baseline is not an analytic procedure, but an empirically determined control condition that can be generically labeled the "double-energy" condition. In the double-energy condition, the stimulus energy from the two monocular stimuli are combined and presented to one eye. The performance obtained with the augmented stimulus is then used as a baseline for evaluating binocular performance. The logic underlying this approach is that in the binocular condition each monocular pathway delivers $x$ (amount of) energy to a common central locus; therefore, the binocular system has available $2 x$ energy for carrying out the binocular task. If this energy is utilized with perfect efficiency, the binocular performance should equal the performance obtained when $2 x$ energy is presented to a single eye. In effect, the double-energy monocular condition serves as an empirical estimate of the summative power of the binocular system if complete, or linear, summation between the monocular pathways is the operating characteristic of the system.

The use of the double-energy monocular condition as a baseline for evaluating summation effects observed under the binocular condition involves the very strong assumption that doubling the energy in one eye is equivalent to delivering one-half of that energy to each eye simultaneously. In practice, it may be difficult for these two methods of presentation to deliver equivalent amounts of energy to a common central locus. An obvious example would be an increment detection experiment where the duration of the stimulus under the double-energy condition had to be increased beyond the critical duration. Appropriate adjustment of parameters could easily remedy this problem. But a less tractable reason why the energy delivered to the common central locus may not be equivalent for the binocular and double-energy conditions is that small nonconjugate eye movements make it difficult for each retina to be stimulated in precisely the same spatial position under the binocular condition. The difference in spatial position between the eyes would act to distribute the energy flowing in the binocular system in a manner different from that under the double-energy monocular condition, and it is likely that this spatial distribution would tend to reduce the effective amount of energy for the binocular condition relative to the double-energy monocular condition. Under these circumstances, the binocular performance would likely be less than the performance observed under the double-energy monocular condition.

Even though practical problems in achieving energy equivalence between the double-energy condition and the binocular condition could be overcome with some effort (e.g., image stabilization), the intrinsic organization of the visual system may preclude such equivalence. For instance, monocular processing would appear to have no counterpart to the potential for areal summation at cortex provided by the binocular case.

These considerations must be taken into account in interpreting the results of the double-energy condition. Unlike the baseline provided by probability summation, performance under the double-energy condition cannot unequivocally be used as a standard for evaluating performance under the binocular condition. Depending upon the experimental context, it may be unreasonable to expect binocular performance to approach that observed for the double-energy condition. Nevertheless, the information provided by the double-energy condition can be quite useful in that it offers one way to estimate the maximum amount of neural summation the binocular system could be expected to yield.

Now that the boundary conditions that govern the interpretation of the results of binocular summation experiments have been described, the next step is to consider the experiments themselves.

\section{BINOCULAR SUMMATION AT THRESHOLD}

The sensitivity measure that has been employed most often in research on binocular summation is the absolute threshold. ${ }^{2}$ The general procedure in most of these experiments has been to present a small light flash to the fovea or the periphery of one or both eyes and 
determine the minimum luminance and/or duration for detection of the flash. Despite the apparent similarity among the various experiments, results from them have been equivocal: some have found the binocular threshold to be lower than the monocular threshold, while others have failed to find such a difference. Moreover, those experiments that have found binocular superiority have found it in different amounts, and consequently have disagreed on the mechanism responsible for summation.

Initially, interest in the problem of binocular summation of the absolute threshold was incidental to the more general problem of dark adaptation in the fovea and the perıphery, for although the existence of a duplex retina, characterized by the anatomically distinct rods and cones, had been established, uncertainty still existed regarding the time course of dark adaptation in the two regions of the retina. Certainly, the different ratios of receptors and ganglion cells in the fovea and periphery presaged the possibility of differences in visual function between the two regions. So it was in this context that the early binocular summation experiments were conceived.

One of the earliest and most widely cited reports concerning binocular summation of the absolute threshold was one by Piper (1903), who measured monocular and binocular thresholds in the peripheral retina at various times during the course of dark adaptation. For the first $15 \mathrm{~min}$ of dark adaptation, Piper found virtually no difference between monocular and binocular sensitivities, which were defined as the reciprocals of the threshold intensities, a convention common among early workers. But after $15 \mathrm{~min}$, the two sensitivity curves diverged, until, at the end of $1 \mathrm{~h}$ of dark adaptation, the binocular sensitivity was approximately one-half the monocular sensitivity. Piper then employed the following reasoning. Since evidence for binocular summation was found only after $15 \mathrm{~min}$ of dark adaptation, and since it was assumed that only the rods were operative by this time, he concluded that binocular summation was solely a function of the rods and consequently a phenomenon limited to the peripheral retina.

Piper's work subsequently was challenged on both logical and empirical grounds. Several workers (Cook, 1934; Graham, 1931; Hecht, 1921) questioned Piper's convention of plotting the reciprocal of threshold intensity as a measure of sensitivity, asserting that such a convention made the unwarranted assumption that the threshold is the inverse of sensitivity. Cook argued that the shape of the sensitivity curves was due to mathematical properties peculiar to a series of reciprocals rather than to basic visual processes. Graham reanalyzed data from Piper's experiment, plotting log threshold intensity against dark adaptation time. This reanalysis not only altered the shape of the curves, but also reduced the difference between the monocular and binocular curves to a degree that Graham concluded was within the range of experimental error. Empirical criticism of the experiments was made by Hecht and Graham, who argued that the failure to control the interval between successive threshold measures during the course of dark adaptation rendered Piper's results untenable.

Although the results from Piper's experiment were discounted by later workers, his data did serve to sustain interest in the problem of binocular summation and its relation to dark adaptation. Recall that Piper's denial of foveal summation was based solely on indirect evidence, i.e., thresholds measured in the peripheral retina. It remained for Abney and Watson (1916) to obtain direct evidence. These authors measured monocular and binocular thresholds for the detection of a small $(1 / 2 \mathrm{deg})$ colored disk. Threshold measurements were begun only after $30 \mathrm{~min}$ of dark adaptation. No evidence for summation was found in the fovea or the area $5 \mathrm{deg}$ vertical from the fovea. These results were replicated in two subsequent experiments by Graham $(1930,1931)$, who found no consistent difference between monocular and binocular thresholds at various stages of dark adaptation.

As mentioned earlier, dark adaptation in the fovea had been denied by some. This issue was not completely resolved until a convincing demonstration of foveal adaptation by Hecht (1921) revealed that the time course of dark adaptation in the fovea was extremely rapid, with the most sensitive state assumed after only a few minutes. This finding led Cook (1934) to reconsider the issue of foveal summation, since the previous experiments concerning foveal summation (i.e., those by Abney and Watson and by Graham) had measured thresholds only after $30 \mathrm{~min}$ of dark adaptation. In a carefully designed experiment, Cook measured foveal threshold for left, right, and both eyes at stages of dark adaptation ranging from a few seconds to nearly an hour. Elaborate efforts were made to insure foveal stimulation and to provide a consistent degree of light adaptation before initiating the dark adaptation period. The procedure employed to measure thresholds depended on the length of the dark-adaptation period. For periods longer than $30 \mathrm{sec}$, the $\mathrm{O}$ maintained foveal fixation until the stimulus target, whose intensity was gradually increased from a subthreshold value, first became perceptible, at which time the $O$ signaled detection of the target. For dark-adaptation periods less than $30 \mathrm{sec}$, the intensity of the target was fixed at a predetermined value; in this case, the dependent measure was time required for detection of the target, with the independent variable being the intensity of the target. By measuring the time needed to detect the target at various intensities, an estimate of the time course of foveal dark adaptation during the first $30 \mathrm{sec}$ could be obtained. Extensive data from two Os revealed that the binocular threshold was, on the average, $50 \%$ lower than that for either eye separately, with this difference being greatest at the shortest dark-adaptation times. Of the results from four other Os, who contributed fewer 
observations, one consistently showed summation while the other three showed variability in both the occurrence and the degree of foveal summation.

As pointed out previously, many of these experiments were actually incidental to the more general problem of dark adaptation. Still, although results from the earlier work on summation of the absolute threshold did not provide conclusive evidence about the occurrence of summation, they did generate interest in the problem and consequently led to experiments concerned solely with binocular summation. In most cases, later experiments were concerned with one of two general issues: (a) discovery of the mechanism responsible for binocular summation, and (b) discovery of the variables influencing the occurrence and magnitude of binocular summation. The remainder of this section will be devoted to the consideration of each of these issues.

\section{Mechanisms of Summation}

\section{Probability Summation}

The notion of probability summation originally was elaborated within the context of the more general problem of the locus of the physiological events critical to the threshold response. Traditionally, this issue has been described in terms of peripheral or photochemical processes vs central processes. Crozier and Holway, proponents of the central hypothesis, argued that the existence of binocular summation of the absolute threshold (Crozier \& Holway, 1939a) and the increment threshold (Crozier \& Holway, 1939b) was evidence for a central locus of the threshold response. But, as Pirenne (1943), a proponent of the peripheral hypothesis, pointed out, it is not necessary to invoke neural summation between the eyes to account for the superiority of binocular vision. As elaborated in the introduction, one would expect the binocular threshold to be lower than the monocular simply from statistical considerations. In support of this argument, Pirenne demonstrated that the increase in the probability of detecting a brief light flash presented to the dark-adapted retina under conditions of binocular viewing is equal to that predicted by the classical probability theorem of independence. In addition, Pirenne reasoned that if there was complete physiological summation between the two eyes, then the binocular probability of detection should equal the monocular probability for detection of a flash of twice the luminance, since the energy entering the visual system is analogous in both conditions. But, contrary to this prediction, he found the double-luminance monocular condition produced a probability of detection that was significantly higher than the binocular probability, a result which precluded complete physiological summation. Rather, he felt that "the outcome of the experiments would be the same if two eyes belonged to different persons, the flash being seen when at least one of the persons has seen it [p. 699]."

Thus, the superiority of binocular vision at threshold does not unequivocally argue for a central locus of the events critical for threshold. Evidence does exist, however, that renders the probability summation model untenable as an appropriate account of binocular summation. Specifically, the probability model predicts tha the degree of summation should remain invariant with temporal or spatial separations of the light flash to each eye, at least within the limits of temporal and spatial variations in retinal sensitivity. But that this is not the case is clearly demonstrated in two experiments, one by Matin (1962) and one by Battersby and Defabaugh (1969). Each will be considered separately.

In an elegant series of experiments, Matin (1962) used a method of constant stimuli to measure the probability of detecting a 2-msec, 35-min circular light flash presented to the dark-adapted periphery of one or both eyes. For binocular stimulation, the interval separating the flashes to the eyes was varied over a large range. Matin was extremely careful to insure that the flashes fell on corresponding retinal locations. Results from a large number of observations using two Ss clearly demonstrated that the probability of detecting the light flash during binocular viewing was dependent upon the interval separating the two flashes. For both Ss, intervals less than $100 \mathrm{msec}$ produced a binocular probability of detection that was greater than the value predicted by the classical probability theorem. For intervals greater than $100 \mathrm{msec}$, the binocular probability was equal to that predicted by probability summation. Matin concluded that the two eyes were not independent detectors and that, rather, neural summation between the two eyes operated at some "common sensory path in the visual system." Matin did point out that although this summation effect was significant, it was considerably less than the summation obtained when both light flashes were superimposed on the same retinal area, a finding which previously had led Pirenne to conclude the absence of complete physiological summation. But Matin offered cogent reasons, based on anatomical and physiological considerations, why an inequality between monocular and binocular summation would be expected. Finally, Matin noted that while the function relating flash interval and probability of seeing for the monocular case (i.e., two flashes presented to the same eye) was unimodal, thus reflecting the well-established time-intensity reciprocity, this function for the binocular case was bimodal, with maximums at flash intervals of approximately 0 and $90 \mathrm{msec}$. This fact, Matin argued, further implicates a neural summation process at a locus more central than the retina.

A strong relationship between binocular summation and temporal separation of the flash to each eye has more recently been found by Battersby and Defabaugh (1969). Using a method-of-limits procedure. they found that the increment threshold for detection of a $5 \cdot \mathrm{msec}$ 
test stimulus presented to one eye was significantly decreased when paired with a 5 -msec subthreshold flash presented to the corresponding area of the other eye. This decrease in threshold was limited, however, to flash intervals of less than $50 \mathrm{msec}$. Furthermore, these authors found that summation between the two eyes was obtained only when the two flashes fell on corresponding retinal areas, a finding consistent with earlier work by Shaad (1935).

The finding that summation is intimately related to temporal and spatial variations in the presentation of light flashes to each eye clearly indicates that probability summation alone cannot account for the greater sensitivity that obtained with binocular viewing. Rather, these results argue strongly for the operation of neural summation between the two eyes. This conclusion receives further support from experiments in which the obtained summation was in excess of that predicted by various normative models. For example, both Collier (1954) and Collier and Kubzansky (1958) have found the difference between monocular and binocular thresholds for the detection of a briefly presented peripherally located flash to be greater than that predicted by the conservative classical probability theorem.

Using the integration model of signal detection theory, Kristofferson demonstrated summation in excess of probability (in Green \& Swets, 1966). Specifically, he obtained monocular and binocular values of $d^{\prime}$, using a four-alternative forced-choice procedure. For both foveal and peripheral stimulation, the obtained binocular values of $d^{\prime}$ were greater than those predicted by the integration model and were more appropriately described by the simple addition of the two monocular values of $\mathrm{d}^{\prime}$. Finally, a recent paper (Westendorf, Blake, \& Fox, 1972) compared binocular performance on a temporal forced-choice detection task with the predictions from two probability models. Brief foveally viewed increments were presented to the eyes either simultaneously or with a 100 -msec onset asynchrony. With simultaneous presentation, binocular detection performance exceeded monocular by an amount greater than that predicted from either the state or the integration models; it was only with asynchronous stimulation that binocular performance fell to the level predicted by these models. This same pattern of results has been observed for decrement detection thresholds as well (Westendorf \& Fox, unpublished observations).

To summarize, there is strong evidence for rejecting the hypothesis that probabilistic considerations alone are sufficient to account for the superiority of two eyes over one. Instead, it is necessary to conclude that this superiority results from some form of neural interaction between the two eyes. In the next two sections, we consider experiments whose results lead to interesting speculations about the nature of this interaction.

\section{Areal Summation}

It is well established that, over a range of stimulus areas, the absolute threshold is related to the size of the test target. While this relationship between threshold and area is complex and depends upon the shape (e.g., Graham, Brown, \& Mote, 1939; Kincaid, Blackwell, \& Kristofferson, 1960) and retinal locus (Graham, 1934) of the target, three general laws seem to describe this relationship approximately (Bartlett, 1965): (a) for small stimulus sizes, threshold is inversely related to the area of the test target; (b) for intermediate sizes, threshold is inversely related to the square root of the area; (c) for large stimulus sizes, luminance alone determines the threshold.

An obvious outcome of binocular viewing is that the total area of retinal illuminance is double relative to monocular viewing. It is not surprising, therefore, that various authors have suggested that the stimulation of corresponding points is functionally equivalent to doubling the area of stimulation in a single eye. Indeed, evidence supporting such a hypothesis was reported by Lythgoe and Phillips (1938). These authors found that at all stages of dark adaptation, the relationship between monocular and binocular thresholds for detection of a $12 \mathrm{deg} 30 \mathrm{~min}$ test patch presented to either the fovea or the periphery was best expressed by the equation

$$
\text { 1.4 } \mathrm{Th}_{\mathrm{b}}=1 / 2\left(\mathrm{Th}_{\mathrm{l}}+\mathrm{Th} \mathrm{h}_{\mathrm{r}}\right)
$$

where $T h_{b}$, $T h_{1}$, and $T h_{\mathfrak{r}}$ are the binocular, left eye, and right eye thresholds, respectively. Lythgoe and Phillips then demonstrated that this equation was reducible to Piper's law. Assuming that binocular observation of a test patch is equivalent to monocular observation of a patch of twice the area, it follows that

$$
\sqrt{2}=\frac{T_{m}}{T h_{b}}, \text { or } 1.4 T_{b}=T h_{m}
$$

where $\mathrm{Th}_{\mathrm{m}}$ is the monocular threshold. This last expression is equivalent to the first when $T_{h}$ is replaced by the mean of the thresholds for the right and left eyes. The authors therefore concluded that the binocular threshold was equivalent to a monocular threshold for a test patch of twice the area. This $\sqrt{2}$ ratio of binocular threshold to the average monocular threshold was replicated in subsequent experiments using smaller test patches and measuring at various retinal positions (Collier, 1954; Crozier \& Holway, 1939b).

Since binocular viewing is functionally equivalent to doubling the area of a monocularly viewed stimulus for threshold observations, one would anticipate that insight into the processes responsible for binocular summation 
could be gained by considering the processes responsible for areal summation. But the problem of areal summation, which, like probability summation, is involved in the more general issue of events critical to the threshold response, has proved difficult to resolve and consequently is beyond the scope of this paper. It is sufficient to say that attempts have been made to account for areal summation either in terms of hypothetical neural interaction among the excitable elements that underlie the threshold response (e.g., Crozier \& Holway, 1939b; Graham, Brown, \& Mote, 1939) or in terms of statistical sampling from a population of units with differing thresholds (Wald, 1938).

\section{Anatomical Considerations}

A series of experiments by Wolf and Zigler (1959, 1963, 1965; Zigler \& Wolf, 1958) has led to an interesting hypothesis concerning the neural events underlying binocular summation. In all these experiments, an ascending-and-descending method of limits was used to measure luminance thresholds for the detection of a small $40 \cdot \mathrm{msec}$ test target presented to various retinal locations. Wolf and Zigler consistently found binocular thresholds to be lower than the threshold for either eye alone when the test target, regardless of shape, fell on corresponding retinal areas on either side of the vertical meridian that bisects the retina. When the test target was centered on the vertical meridian, the occurrence of binocular summation depended on the extension of the target into areas lateral to the vertical meridian. Specifically, the binocular threshold was no lower than the monocular threshold when a small square target or a thin rectangular, vertically oriented target fell on the vertical meridian. But when the rectangular target was oriented horizontally on the vertical meridian, extending into areas lateral to this meridian, summation was found. An especially curious finding reported by these authors was that a slight displacement of the image of the vertically oriented rectangle in one eye to one side of the meridian reestablished binocular summation, despite the fact that this image displacement produced stimulation of noncorresponding retinal areas. But a similar displacement of the image in one eye in the lateral periphery did not result in summation.

These results led Wolf and Zigler (1965) to propose the following hypothesis: Due to the partial crossing over of the optic nerve fibers in man, fibers from the nasal portion of each retina terminate in the contralateral hemisphere while the fibers from the temporal portion terminate in the ipsilateral hemisphere. Thus, when a binocularly viewed test target falls on corresponding retinal areas that are lateral to the vertical meridian, the resulting impulses from each eye travel to the same hemisphere, reinforcing each other and producing an unequal distribution of excitation between the two hemispheres. But when the test target falls on the vertical meridian of each eye, parts of the visual image fall on each half of the retina and impulses are carried to both hemispheres. The result, they assert, is an equal and weaker excitation of each hemisphere. The authors conclude, therefore, that binocular summation can be produced either by reinforcement of excitations from corresponding retinal halves or by an unequal distribution of excitation between the two hemispheres.

The precise nasotemporal division assumed by Wolf and Zigler does indeed exist. It has been shown by retrograde degeneration that the separation of ipsilateral and contralateral fibers along the vertical meridian results in a cleavage line that clearly transects the center of both foveae (Polyak, 1957, p. 330). Thus, it seems reasonable that a test patch falling on corresponding points of the vertical meridian in each eye could result in a distribution of neural impulses to postchiasma areas that is different from the distribution resulting from a test patch falling on corresponding points lateral to this meridian. And assuming that the binocular threshold response is determined by the distribution of neural impulses in postchiasma areas, one could anticipate differences between the two binocular thresholds. But, in applying the same argument to monocular thresholds, it is not clear why the monocular thresholds do not reflect similar differences; that is, a single target on the vertical meridian of one retina should result in a distribution of neural impulses to postchiasma areas that is different from that of a monocular target that falls to either side of the vertical meridian. And, since the criterion for the existence of binocular summation is a difference between binocular and monocular thresholds, binocular viewing should maintain its relative efficiency over monocular viewing despite an increase in threshold for both. But this was not the result in the experiments by Wolf and Zigler. While the binocular threshold measured on the vertical meridian was elevated, the monocular threshold along this same meridian was virtually unaffected. Thus it would appear that the hypothesis requires further clarification.

Despite the questions raised about the theoretical account of binocular summation offered by Wolf and Zigler, the fact remains that the results from their carefully performed experiments are impressive. Furthermore, their results perhaps can account for the earlier failure of Abney and Watson (1916) to find evidence for summation in the fovea or in the periphery. In their experiments, a 34-min target was positioned on the vertical meridian which extended from the point of foveal fixation $5 \mathrm{deg}$ into the periphery. And it is along this meridian that Wolf and Zigler also failed to find evidence for summation. The negative finding of Abney and Watson possibly is explained by their selection of the vertical meridian as the area of stimulation.

\section{Variables Affecting the Occurrence of Binocular Summation}

In spite of the convincing evidence for binocular 
summation of the absolute threshold, the fact still remains that a substantial number of Es have failed to obtain evidence for summation (Abney \& Watson, 1961; Bartlett \& Gagné. 1939: Downey, 1919; Graham, 1930). In the following section, an attempt is made to identify various factors that possibly influence the occurrence and magnitude of binocular summation.

\section{Individual Differences}

The literature concerning visual perception is replete with examples of wide individual differences on various tasks. Moreover, it is well established that humans exhibit varying degrees of ocular dominance (Walls, 1951). There is, then, certainly no a priori reason to expect homogeneity between individuals concerning the degree of binocular summation. On the contrary, it is possible that summation occurs only if the two eyes of an individual are relatively equal in sensitivity; if the two eyes are markedly different in sensitivity, then the more sensitive could dominate during binocular viewing.

In some of the experiments purporting to demonstrate the absence of summation between the eyes, inspection of individual data included in the report reveals that, contrary to this general conclusion, some Ss do show a summation effect. An example of this is an experiment by Graham (1930), in which he used an ascending method of limits to measure monocular and binocular thresholds in the fovea at various times during the course of dark adaptation. In describing the results, Graham reported individual data from two Os who showed "the greatest variation from the average [p. 502]" along with the combined or averaged data for nine Os, including the two individuals. Inspection of the individual results (Table 2, p. 503) reveals that, for ST.H., the binocular threshold was lower than the monocular for 6 out of 10 values of dark adaptation, a result consistent with Graham's conclusion of no summation. For the other S, M.N., the binocular threshold is lower in 8 out of 10 cases. Graham argued that although the results of M.N. apparently reflect a slight summation effect, this difference is "well within the limits of experimental error" (no inferential statistics were offered in support of this claim) and can "be discounted when it is shown that other observers showed the reverse tendency [p. 502]." Indeed, inspection of the average monocular and binocular thresholds for all nine Ss (Table 3, p. 505) reveals no evidence for summation in the fovea. And, according to Graham, averaging the results of a number of Os has "the advantage of presenting the theoretical processes [p. 504]." The strategy, however, of averaging the data from a number of $O s$ has been questioned by Crozier and Holway (1939a), who argue that "one source of confusion can be avoided by refraining from the practice of averaging measurements from a number of individuals. . . . In the absence of true homogeneity mere increase of superficial statistical precision may serve only to obscure the fundamental phenomena one is seeking to explore [p. 344]."

It should be pointed out that, in a subsequent experiment, Graham (1931), using apparatus and procedures similar to those used in his earlier experiment, failed to find evidence for summation in the peripheral retina. For none of the six individuals was the binocular threshold consistently lower than the monocular; in fact, there was a surprisingly large number of paradoxical cases where the monocular threshold was lower than the binocular!

Another example of the practice of drawing conclusions from data averaged over $S$ s is a series of experiments by Bartlett and Gagné (1939). Working under the direction of Graham, these workers used an ascending and descending method of limits to obtain intensity thresholds for the detection of an 8 -msec light flash of $1 \mathrm{deg}$ of visual angle. Results from three experiments, in which both natural pupils and artificial pupils were employed, led the authors to conclude that there was not a significant difference between the binocular threshold and the threshold associated with the more sensitive eye. However, no inferential statistical support was included. Inspection of Table 2 (p.97) in their article reveals that for 6 of the $10 \mathrm{Ss}$ the binocular threshold was lower than the best monocular threshold.

The primary purpose of discussing these experiments has been to demonstrate that the existence of binocular summation may not be universal, and, therefore, conclusions as to its operation under particular conditions should not be based on data averaged over Ss. The important point is that the relative efficiency of binocular viewing is subtle and differs between individuals, a fact that strongly argues for the use of inferential statistics to supplement any conclusion concerning the existence of summation.

\section{Pupil Size}

An essential condition for the comparison of monocular and binocular thresholds is that the amount of light reaching the retina during monocular viewing be the same as the total amount reaching each retina during binocular viewing. But it is known that pupil diameter during binocular viewing is smaller than during monocular viewing (Reeves, 1918; Thomson, 1947), and, since the amount of light enterning the eye is proportional to the square of the diameter of the pupil, this difference in pupil size could differentially affect the monocular and binocular thresholds and consequently vitiate any test for summation. Specifically, the large pupil size during monocular viewing could lower the monocular threshold and mask any summation effect. These facts, then, argue strongly for the utilization of artificial pupils, preferably no larger than $2 \mathrm{~mm}$, since pupil diameter during medium illumination is typically $2-3 \mathrm{~mm}$ (Reeves, 1918).

But, while it is clear that variations in pupil size can 
differentially affect the monocular and binocular thresholds, it appears that such variations are not solely responsible for the lack of agreement concerning summation of the absolute threshold, for a number of Es who have found evidence for summation have employed artificial pupils (e.g., Forbes \& Mote, 1956; Laird, 1924) while others who have employed artificial pupils have failed to obtain such evidence (e.g., Graham, 1930).

\section{Fixation}

Since the studies of binocular summation of the absolute threshold have been concerned with presenting small spots of light to specific retinal areas, the necessity for accurate fixation is obvious and consequently has received careful attention from a number of workers. But simply providing a clear fixation point is not sufficient. The fixation point and the test target should be on the same horopter in order to insure proper accommodation and to promote proper convergence for the stimulation of corresponding retinal points, a condition apparently necessary for the operation of summation (Shaad, 1935).

In virtually all binocular summation experiments, the monocular threshold has been obtained by occluding one eye; fixation during these measurements is necessarily monocular. But, when obtaining binocular thresholds, the fixation is always binocular. In order to determine if these different conditions of fixation contribute to the difference between these two thresholds, Collier (1954) measured both monocular and binocular probability-of-seeing thresholds during both monocular and binocular fixation. Results from his experiments revealed that binocular fixation produced a slight improvement in monocular performance relative to the condition of monocular fixation, but that this improvement was not great enough to eliminate the difference between the monocular and binocular thresholds, which, for all conditions, was greater than that predicted by the classical probability theorem of independence. An interesting result from these experiments was that the binocular threshold was significantly elevated when only one eye was used for fixation. A likely explanation of this result is that nonconjugate eye movements of the unfixated eye resulted in stimulation of noncorresponding retinal points, a possibility considered by Collier.

\section{Method of Stimulus Presentation}

Virtually all the experiments that have assessed summation of the absolute threshold have used either percent correct or intensity of a just perceptible target as estimates of threshold. [Two notable exceptions are the experiments by Cook (1934) and Downey (1919), both of whom used the time required from onset of dark adaptation to detect a dim light as the measure of threshold; the former found evidence for summation in the fovea, while the latter failed to find such evidence.] Typically, the percent-correct estimate has been obtained with a method of constant brightness, i.e., a briefly presented target of fixed intensity, whereas the minimum intensity threshold has been obtained by some form of the method of limits, i.e., a systematic increase and/or decrease in the luminance of a target of duration sufficient to be easily seen at higher luminances.

It is interesting to note that three of the failures to find evidence for summation employed dim targets that remained on until the $\mathrm{O}$ reported detection (Downey, 1919; Graham, 1930, 1931). As described earlier, in Graham's experiments the intensity of a small target was gradually increased until the $O$ reported detection. In Downey's experiment, a dim light of constant luminance was continuously present and Os reported when the light was first perceived. The possibility is raised, then, that this method of stimulus presentation does not produce a large summation effect. It seems reasonable that the neural response to a light that is always present but simply made more intense would be different from the neural response to a brief flash of light. Thus, summation may be significant only for the latter condition. However, this hypothesis fails to explain why Cook (1934) did obtain evidence for binocular summation even though the continuous stimulus presentation method was employed, or why Bartlett and Gagné (1939) failed to find evidence for summation with the brief illumination method. Furthermore, this hypothesis is contrary to one offered by Bartlett and Gagné, who suggested that a possible condition for the occurrence of binocular summation is the use of long repeated exposures. Their hypothesis was based on Cook's findings.

A more recent experiment by Collier and Kubzansky (1958) demonstrates that both the method of constant brightness and the method of limits are capable of producing summation beyond that predicted by probability. They did find, however, that the magnitude of the summation effect was significantly less for the method of limits.

\section{Sensory vs Criterion Factors}

The fact that the method of stimulus presentation can affect the obtained threshold value raises the distinction between sensory processes and decision criterion. The latter term refers to nonperceptual factors, such as the willingness of Os to report the detection of the target stimulus. Only recently has this distinction received emphasis and efforts made to separate the criterion factor from the sensory process. Experiments investigating binocular summation have often employed some form of the simple yes-no indicator response. And. as Swets (1961) has pointed out. restricting the $O$ to these two response categories does not permit an estimate of sensitivity independent of decision criterion. Thus. the lack of empirical agreement concerning the 
existence of binocular summation could be due, at least in part, to differences in instruction, motivation, etc., among the various experiments (see Pirenne \& Marriot, 1959). Furthermore, a difference between monocular and binocular performance could possibly reflect the fact that, during binocular viewing, an $\mathrm{O}$ is more willing to report the detection of a test stimulus. This is, of course, the basis of the multistate model of independence described in the introduction. It is unlikely, however, that a decision-criterion effect is solely responsible for the superiority of binocular viewing, as evidence for binocular summation has also been obtained using forced-choice methods which are generally not susceptible to response biases.

\section{Summary}

While early Es were in disagreement concerning the existence of binocular summation of the absolute threshold, more recent Es have consistently found the binocular threshold to be lower than the corresponding monocular threshold. Furthermore, the relative superiority of binocular viewing is greater than that expected on the basis of probability summation. Finally, it was demonstrated that while certain variables, such as pupil size and fixation, can influence the magnitude of the difference between monocular and binocular thresholds, these variables are not solely responsible for this difference.

\section{BINOCULAR BRIGHTNESS SUMMATION}

This section is devoted to an examination of the difference in the brightness of suprathreshold stimuli as a function of binocular and monocular viewing, an issue referred to as binocular brightness summation. The simplest experiment of this type, which the reader can easily perform, is to note the brightness of some object using both eyes, and then note if the brightness of the object appears diminished. The experiment reveals that binocular viewing does not produce any dramatic increase in perceived brightness; clearly, there is no doubling of brightness. Nevertheless, it is reasonable to suppose that there may be differences in monocular and binocular brightness, but that such differences would be detected only by sensitive experiments. Relatively few experiments have been addressed to the issue of binocular brightness summation, and the results are equivocal. It should be noted that there are certain classes of experiments that involve procedures resembling those used in brightness summation experiments but are conducted for different purposes; some examples are the measurement of retinal light adaptation (e.g., LeGrand, 1967) and simultaneous contrast (Whittle \& Challands, 1969). In such experiments, interocular brightness matches are made in order to eliminate retinal interaction between test fields. When these kinds of experiments are excluded from consideration, the number of experiments directed to the question of binocular brightness summation is not very large.

The majority of the early work on binocular brightness centered around the phenomenon known as Fechner's paradox, first described by Fechner (1860). This phenomenon occurs when the two eyes are unequally illuminated, as would be the case if one eye viewed an object through a neutral density filter while the other eye viewed the object directly. Under these conditions, binocular brightness is less than that observed with the uncovered eye alone; thus, closing the filtered eye, and therefore reducing the visual stimulation, produces a paradoxical increase in brightness. It is as if the dimmer field subtracts brightness from its brighter partner rather than adding brightness. ${ }^{3}$

An enduring hypothesis that has evolved from observations of Fechner's paradox is that binocular brightness is the product of an averaging process between the two eyes. For example, based on introspective observation, Sherrington (1906) concluded that binocular brightness was "somewhat, but not far, above the arithmetic mean of the values of the two uniocular components as expressed by the measures of the physical stimuli yielding them [p. 373]." This conclusion proved true both for a light flickering above threshold and for a steady, unflickering light. Sherrington did cite cases where the brightness of a binocularly viewed target appeared brighter than the same target viewed monocularly, but suggested that this summation effect might be due to superior accommodation and convergence during binocular viewing or to an attenuation of monocular brightness by the darkness in the other eye.

In a subsequent experiment, Dawson (1913) also reported that the brightness of an object did not differ as a function of monocular and binocular viewing, a prediction that follows directly from a simple brightness averaging model. Interestingly, $O s$ in Dawson's experiment were quicker and more accurate in locating a dim ring on a uniform background when the display was binocularly viewed, relative to monocular viewing. But rather than a difference in brightness, the Os reported that binocular viewing produced a steadier, more persistent image of the dim ring. In an attempt to account for the relative superiority of binocular viewing and yet remain consistent with the introspective reports of his Os, Dawson concluded that brightness summation was not operative and that the superiority of binocular viewing was due to the increased clarity of the binocular image. This increased clarity was attributed to the independent development, fluctuation, and utilization of the two monocular images, a conclusion undoubtedly influenced by Dawson's contemporary, C. S. Sherrington.

Up to this point, introspection had failed to reveal any evidence for brightness summation between the two 
eyes. This apparent lack of brightness summation puzzled DeSilva and Bartley (1930), who cited both anatomical and phenomenological reasons why one would expect the eyes to function integratively in producing the binocular impression of brightness. Moreover, these authors were skeptical of conclusions based on phenomenal reports, since this method, they argued, was liable to considerable inaccuracy. As an alternative, they favored a brightness-matching procedure whereby a $S$ matched the brightness of a test target to that of a comparison target. With this procedure, it was not necessary to rely on memory, since the $S$ could always refer to the comparison target while adjusting the brightness of the test target.

DeSilva and Bartley obtained simultaneous brightness matches using an apparatus with which light from the comparison target to the left eye could be systematically reduced by rotating an episcotister blade. The right eye viewed the comparison target directly, and both eyes viewed the test target directly. The test and comparison targets were initially equated for brightness during direct binocular regard. Next, the episcotister was used to decrease the luminance of the comparison target to the left eye, and once again, the two targets were equated for brightness. DeSilva and Bartley found that the brightness of the test target invariably had to be reduced in order to produce a brightness match. When all light from the comparison target to one eye was occluded, the brightness of the binocular test target had to be reduced as much as $44 \%$ to match the brightness of the monocularly viewed comparison target. This latter result indicates a brightness summation effect, and it was observed using a number of different luminance values, target sizes, and degrees of target separation.

In a subsequent experiment, Fry and Bartley (1933), using apparatus and procedures similar to those described in the previous experiment, also found evidence for brightness summation. Serving as their own Ss, they viewed two small rectangular targets separated horizontally by $1 \mathrm{deg}$. The luminance of the top target, which was monocularly viewed, was set at various levels, and at each level the $O$ adjusted the brightness of the binocularly viewed lower target. At all intensity levels measured, the binocular target had to be reduced in brightness in order to obtain an equation with the monocular target, with the reduction as great as $50 \%$ at certain intensities.

Until recently, these last two experiments constituted the main evidence for the existence of brightness summation between the two eyes. Yet some workers have been reluctant to consider these experiments conclusive, arguing that the results could be questioned on procedural grounds. For example, Graham (1931) has pointed out that the lack of pupil control in those experiments is a potential source of error. Another concern voiced by Graham is that the relatively large expanse of darkness between the test and comparison targets in the experiment of DeSilva and Bartley could be expected to produce poor discrimination. Finally, more recently, Levelt (1965) has argued that matching monocular and binocular brightnesses is an unstable affair, subject to high between-session variability.

The most recent comparison of monocular and binocular brightness has been reported in an experiment by J. C. Stevens (1967). He required Ss to give magnitude estimates to each of seven different luminance settings of a target viewed by one eye and by both eyes. Consistent with previous research using magnitude estimates, the resulting relationship between luminance and brightness estimates was accurately described by the psychophysical power function. But inspection of the monocular and binocular functions revealed that they were virtually identical, differing by 1 jnd. Stevens felt that even this slight difference might be due to the fact that during a monocular presentation the unused eye had additional time to dark-adapt. Stevens did not dismiss completely, however, the possibility of summation, pointing out that the method of magnitude estimates may lack the precision to reveal a summation effect that under certain conditions has proved to be small but real.

Since stimulus brightness generally is highly correlated with some measure of its physical intensity, such as luminous flux, brightness can often be scaled indirectly by some response sensitive to physical intensity. Reaction time (RT) is an appropriate response, since the relationship between $\mathrm{RT}$ and physical intensity is inverse (e.g., Woodworth \& Schlosberg, 1954).

In view of this relationship, it is somewhat surprising that RT has not been used more frequently to investigate binocular brightness summation. As far as we can determine, only two experiments have addressed this question directly. There is the widely cited paper by Poffenberger (1912), who concluded that RT under binocular viewing was faster than for monocular. More recently, Minucci and Connors (1967) systematically examined the relationship between monocular and binocular simple RT to a brief flash of light varied in intensity over a 4-log-unit range. RT was a negatively accelerated function of increasing intensity, and, for all points, binocular RT was reliably faster than monocular RT. From these data, the authors concluded that the increase in binocular RT was greater than that anticipated from complete summation. What is not clear, however, is the method they used to establish the baseline from which the magnitude of summation could be determined.

This difficulty may point up a general problem associated with the use of RT to assess binocular summation. The superiority of two eyes over one on RT tasks, just as on detection tasks, could be attributed either to neural summation or to probability summation. But, unlike the case with detection, it is not obvious how to establish the level of RT performance expected on the basis of probability summation. One strategy might be to convert the latency measures to probability 
values using one of several stochastic latency models (e.g.. see McGill, 1963). From these conversions, probability summation models could then be developed. One prediction that would follow from any such model is that at asymptote binocular and monocular RTs should be equivalent, since the asymptotic values correspond to a probability level approaching 1.0. On the basis of neural summation, however, one would predict that binocular latency, even at asymptote, would be less than the asymptotic monocular latency. The problem in evaluating these two alternative predictions is that asymptotic RT levels were not obtained by Minucci and Connors, and frequently such levels are not readily obtained in RT experiments. Thus, while the existence of faster binocular RTs has interesting and even practical implications, its theoretical relevarice remains equivocal.

\section{Binocular Brightness Models}

Having summarized the few experiments concerning brightness summation, consideration will now be given to three alternative models of binocular brightness.

\section{Fry and Bartley Model}

Obviously, the variety of binocular brightness phenomena, ranging from brightness summation to brightness subtraction (i.e., Fechner's paradox), poses a formidable problem for any theory of binocular brightness. Nevertheless, Fry and Bartley (1933) concluded that these seemingly contradictory results are compatible if one assumes that both summation and inhibition processes are operative in producing the final binocular brightness. The summation process is accomplished, they hypothesized, by the convergence of impulses from corresponding retinal areas onto a common pathway in the brain. This convergence results in a physiological summation of neural impulses. The inhibitory process is due to the depressing effect of a border contrast in one eye on the activity from the corresponding area of the other eye, a notion originally developed by Hering. Thus, the final binocular brightness is more brilliant than either monocular impression only when the increased activity due to summation more than compensates for the decrease due to inhibition.

Evidence consistent with this hypothesis has been offered by Fry (1936) and more recently by Leibowitz and Walker (1956). The latter authors found that as the size of a square field was increased from 15 to $60 \mathrm{~min}$, the brightness summation effect, as measured by a brightness-matching procedure, was tripled. This result could be explained, they concluded, by the fact that as field size is enlarged the area increases at a more rapid rate than border length, a condition that would decrease any influence that border contrast has on the brightness of the field. And this result, as Leibowitz and Walker point out, is consistent with the Fry and Bartley model.

\section{Levelt Luminance-Averaging Model}

Levelt (1965) has argued that for the Fry and Bartley model to account for all the various brightness phenomena, new assumptions must be made about the relative influence of the summation and inhibition processes. And, while explanation is possible, the theory is weakened by these new assumptions. As an alternative, Levelt proposed a simpler model that is a contemporary statement of the brightness averaging hypothesis. ${ }^{4}$ On this model, binocular brightness is based on the linear combination of the weighted monocular luminances, such that

$$
W_{L} E_{L}+W_{R} E_{R}=C,
$$

where $C$ is the binocular brightness, $E_{L}$ and $E_{R}$ are the luminances of the fused left- and right-eye targets, and $W_{L}$ and $W_{R}$ are weighting coefficients that, in general, reflect the amount of contour in each eye. The sum of these two weights is unity. This equation states that in order to maintain a constant binocular brightness (C), any increase in luminance to one eye must be accompanied by a decrease in luminance to the other.

The data offered by Levelt in support of this model was obtained by a successive brightness-matching procedure: The luminance of the test target to one eye was set by the $E$, and the $O$ adjusted the luminance of the test target to the other eye until the binocular brightness of the test target was equal to the brightness of a binocular comparison target composed of equal monocular luminances. The test target and comparison target were viewed successively. This procedure produced a set of left- and right-target luminances that produced the same binocular brightness when fused. Levelt refers to a plotting of these various luminance pairs as an equibrightness curve. The equibrightness curves he obtained were described nicely by the luminance-averaging model, except at low values of monocular luminance, where the resulting relationship was curvilinear and, according to Levelt, not predictable. He hypothesized that these curvilinear portions of the curves were due to the peculiar behavior of the weighting coefficients when light to one eye was near threshold.

An interesting observation, and one that Levelt fails to discuss, concerns the adjusted luminance of the test target in one eye when the luminance of the test target in the other eye is zero. Inspection of the equibrightness curves reported by Levelt reveals that under this condition, the luminance of the monocular test target consistently was adjusted to a value in excess of the luminance of the binocularly viewed comparison target, a result implying the operation of brightness summation. But the Levelt model is strictly an averaging model, and since the two weighting coefficients are fractions whose sum is unity, no combination of weighted monocular luminances can yield a binocular brightness greater than 
the more luminous monocular field. Therefore, on the basis of results from Levelt's experiments, one is forced to conclude that the luminance-averaging model is limited to conditions where a nonzero luminance is presented to each eye.

Yet another limitation on the luminance-averaging model has been pointed out by Teller and Galanter (1967). The luminance-averaging model specifies that binocular brightness is based upon the combination of monocular luminances. Indeed, Levelt (1968) has rejected the notion that monocular brightnesses, rather than luminances, are the basis for binocular brightness. But Teller and Galanter found that the binocular brightness of two fused monocular targets of constant luminance was affected by variations in the brightness of one of the two targets even though the luminance of that target was unchanged. This variation in the brightness of a target of constant luminance was accomplished in two ways: (a) the constant-luminance target was presented to one eye at different points of dark adaptation, and (b) the luminance of the background upon which the constant-luminance target appeared was varied, thus inducing brightness contrast. With both these methods. the judgment of binocular brightness was influenced by the variation in the brightness of the target to one eye despite the fact that the luminance of this target was invariant. These results suggest that Levelt's luminance-averaging model is limited to the condition where luminance and brightness are perfectly correlated.

\section{Autocorrelation Model}

An even more recent binocular brightness model proposed by Engel $(1967.1969)$ is an elaboration of the luminance-averaging model. This model, the autocorrelation model, utilizes magnitude estimates of monocular brightness to predict the binocular brightness of the combined monocular fields. The specific expression for the predicted binocular magnitude estimate, $\psi_{\mathrm{B}}$, depends on the procedure used to obtain brightness matches, i.e.. simultaneous vs successive matching, but in general the binocular brightness estimate is a function of the square root of the weighted, squared monocular magnitude estimates, $\psi_{R}$ and $\psi_{L}$.

While the luminance-averaging model and the autocorrelation model have features in common, they also differ in two respects. The first difference is in the operations used to define the weighting coefficients. Levelt did not specify an operation by which these weights could be defined. but simply manipulated their values experimentally by varying the amount of contour in each eye. Engel. however, proposed a specific mathematical operation for determining the weighting coefficient for each eye. The derivation of these weights involved finding the integral of the squared autocorrelation function for the pattern in each eye (see Engel. 1967. 1969. for details). The number resulting from this operation can be considered as a measure of the amount of contour and contrast in that eye. The two numbers associated with the two monocular patterns are then normalized so that

$$
W_{L}^{2}+W_{R}^{2}=1
$$

Thus, the resulting weights reflect the amount of contour and contrast in one eye relative to the other.

The second difference between the luminance-averaging model and the autocorrelation model is in the monocular components assumed to produce the binocular brightness response. As pointed out earlier. the luminance-averaging model asserts that the visual system utilizes the monocular luminances to produce the binocular impression of brightness. But the autocorrelation model asserts that monocular brightnesses, rather than luminances, are combined to produce binocular brightness. Certainly, the experiment of Teller and Galanter would support the latter assertion.

\section{Comparisons of the Models}

Using both simultaneous and successive brightness-matching tasks, Engel (1967) found that the binocular brightness of various pairs of fused monocular targets, most of unequal luminance, was adequately described by both the luminance-averaging model and the autocorrelation model. The amount of contour and contrast in both eyes was identical. Thus, this experiment was unable to differentiate the two models. a result that is not surprising when one considers the similarity of the two models. Indeed. Engel demonstrated this similarity by rewriting the magnitude estimate in terms of luminance, using Stevens's power function for brightness. The autocorrelation model was virtually identical to the luminance-averaging model, the predictions differing by an amount less than the standard error of the observations.

In a recent article, Engel (1969) tested the ability of the autocorrelation model to reproduce results from experiments by Levelt and by Fry and Bartley. These particular experiments were selected because results from them represented apparently conflicting findings: binocular brightness averaging, as suggested by Levelt's data, and summation, as suggested by Fry and Bartley's data. The method used for generating predictions was computer simulation; the mathematical properties asserted by the autocorrelation model to be responsible for binocular brightness were programmed. and the stimulus displays of the various experiments were numerically coded in a manner interpretable by the program. This numerical coding consisted of defining a subset(s) of an $n$ by $n$ matrix as the target(s) and the remainder of the matrix as background. The brightness responses to the hypothetical targets were simulated by assigning to each target a magnitude estimate of the 
actual luminance of the target as reported in the original experiment. These estimates were based on Stevens's power function for brightness (exponent $=0.33$ ). The monocular weights, $W_{L}$ and $W_{R}$ were then derived by translating the autocorrelation function, a continuous function, into a discrete function defined over the elements of the matrix. The magnitude estimates could then be used to derive a number that represented the amount of contour and contrast in the matrix. The mathematical operation presumed to underlie binocular brightness,

$$
\psi_{\mathrm{B}}=\left[\left(\mathrm{W}_{\mathrm{R}} \psi_{\mathrm{R}}\right)^{2}+\left(\mathrm{W}_{\mathrm{L}} \psi_{\mathrm{L}}\right)^{2}\right]^{3 / 2},
$$

was used to generate brightness matches, where $\psi_{\mathbf{L}}$ and $\psi_{\mathbf{R}}$ correspond to the monocular components of the comparison target and $\psi_{\mathrm{B}}$ corresponds to the test target. Brightness matches were simulated by holding two of the values constant while the computer solved for the third value.

Brightness matches from the computer simulation, in general, were in good agreement with the actual results obtained in the various experiments. Two results of the simulation were especially impressive. First, the autocorrelation model faithfully reproduced the nonlinear portions of an equibrightness curve, including the terminal points which, as pointed out earlier, imply the operation of brightness summation. This reproduction is striking when one recalls that Levelt concluded that these portions of the curve were unpredictable. It should be noted, however, that the equibrightness curve reproduced by the autocorrelation model is but one of a number of such curves, many of which display quite dissimilar behavior in the nonlinear portions.

A second striking feature of the results from the simulations was the ability of the autocorrelation model, basically a brightness-averaging model, to produce a result that suggests the operation of brightness summation, i.e., the results from Experiment 1 of Fry and Bartley. Recall that in this experiment, the $O$ adjusted the brightness of a binocularly viewed comparison target until it was judged equal to a monocularly viewed test target located $1 \mathrm{deg}$ below the comparison target. The adjusted luminance of the binocular comparison target was consistently less than the monocular test target, a result implying brightness summation. But, according to Engel, this apparent summation effect was due to the close proximity of the binocularly viewed comparison target and the monocularly viewed test target. As a result of this proximity, the weighting value associated with the darkened test target eye was not zero but, rather, some finite value due to the presence of the comparison target in that eye. And, since the weights for each eye are inversely related, the corresponding weight for the lighted test target eye was less than one. Thus, in order to equate the two targets, the monocular brightness
$\left(\psi_{L}\right)$ had to be greater than the combined binocular brightness $\left(\psi_{\mathbf{B}}\right)$. This argument, then, attributes the apparent brightness summation effect to an artifactual drop in the brightness of the monocular target. It should be pointed out that once again the simulated results correspond to just one particular curve reported by Fry and Bartley and that two other curves reported in their paper, curves ostensibly reflecting the operation of brightness summation, are different in form from the one generated by Engel.

In a subsequent experiment, Engel (1970), using a procedure identical to that of Fry and Bartley, found that as the distance between a monocularly viewed test target and a binocularly viewed comparison target was systematically increased, the difference between the binocular and monocular brightness decreased. But even at the largest separation distance $(2 \mathrm{deg})$, evidence for brightness summation was found. Furthermore, successively presenting the two targets, thus eliminating any spatial contiguity between the two targets, still yielded a brightness summation effect. These results led Engel to conclude that for simple displays "binocular brightness is greater than monocular brightness [p. 350]," but that this difference might be due to the binocular target's appearing sharper, or more clear.

In evaluating the autocorrelation model, one must wonder to what extent the computer-generated results are due to the arbitrary assignment of numbers to the stimulus situations. For example, Engel found it necessary to assign nonzero brightness estimates to the matrix elements associated with black background (and consequently to the dark field of the "monocular" test target in Fry and Bartley's first experiment). Indeed, Engel acknowledged the difficulties associated with implementing the simulations. But these numerical translations are not unrealistic, he argued, in view of certain physiological facts. For example, Engel stated that the spontaneous activity of receptors in the absence of light is compatible with the notion of a finite brightness response to a black background.

The use of magnitude estimates to measure the response of the visual system to brightness is also not without question. One must wonder, as has Stevens (1967), whether or not the method of magnitude estimation possesses the precision to detect summation effects, which often are subtle.

Despite the questions posed in the last few paragraphs, the ability of the autocorrelation model to predict rather diverse brightness phenomena is impressive; certainly, the model merits further consideration as an appropriate theoretical account of the process responsible for binocular brightness. That the model might have even more general applicability is suggested by Engel, who feels that with certain elaborations the model could be considered as a principal mediator of visual pattern processing.

To summarize, the weight of existing evidence seems to suggest that a binocularly viewed surface appears 
somewhat brighter than the same surface viewed monocularly. But it remains to be seen whether this phenomenon is due to a true summation effect between the two eyes or to artifactual conditions peculiar to the brightness-matching tasks typically employed. What is clear is that when the two eyes are presented with unequal luminances. the resulting binocular brightness is a compromise between the two monocular brightnesses. Thus, if the concept of true brightness summation is accepted, one is forced to consider binocular brightness models that incorporate both summation and inhibition components.

\section{FLICKER FUSION THRESHOLD}

The point at which an intermittent flash of light is first perceived as continuous or steady is referred to as the critical flicker frequency (CFF). The classical experiments by Sherrington $(1904,1906)$ are the pioneer work concerning binocular flicker summation and are representative of the rationale underlying most of the later research on binocular flicker sensitivity. The experiments by Sherrington were a logical extension of his research on the integrative action of the central nervous system, in which he had established that various reflex ares converge upon a common path. where they reinforce each other, producing a more robust response (allied reflexes). or inhibit each other. producing an ensuing "struggle for dominance" (antagonistic reflexes). Therefore. "From these motor reactions it is natural to attempt to cross the gulf from movements to sensation

[to] test to what degree the visual singleness ... is due to direct confluence of the sensory paths excited by the right eye and left eve images respectively [1906, p. 356]."

Sherrington reasoned that if the physiological activity from each eye was summed or combined at a neural locus common to the two eyes, then a pattern of intermittent illumination 180 deg out of phase between the eyes would produce a steady level of neural activity at this common locus. In effect, the bright phase in one eye would efface the dark phase in the other, thus drastically reducing or completely eliminating the perception of flicker. Furthermore, Sherrington assumed that if there was physiological summation between the two eyes, then perception of flicker during binocular viewing of in-phase light flashes should persist long after the fusion of flicker with monocular viewing, because the brightness of the left- and right-eye flashes would reinforce each other in the manner of allied reflexes.

To test these possibilities. Sherrington compared binocular simultaneous-flicker thresholds with binocular alternating-flicker thresholds. Sherrington had Os successively view two vertically aligned disks that were flickering at a rapid rate and report which disk first appeared to flicker. One disk was composed of two fused monocular images flickering so that the light and dark phases of each flash occurred simultaneously in both eyes. The other disk was composed of two fused monocular images, flickering so that a light phase in one eye was accompanied by a dark phase in the other. A revolving cylindrical shutter was used to vary the rate of intermittence. The rate was set initially at a level above the CFF and then slowly decreased until the $O$ reported the detection of flicker in one of the two disks. In order to make this judgment, it was necessary to stare successively at one disk and then the other, a task requiring a 3 -deg vertical eye movement.

Sherrington found that the CFF for the binocular simultaneous-flicker condition was slightly higher than for the binocular alternating-flicker condition. Furthermore, the CFF obtained during monocular viewing, which was produced by occluding the image of one disk to one eye, was slightly lower than the binocular simultaneous CFF but higher than the binocular alternating-flicker CFF. But even though these differences were consistent for all Ss, Sherrington felt that the magnitude of these differences, which was only about $3 \%$, was too small to conclude that impulses from corresponding retinal areas interacted physiologically to produce the binocular response to flicker. Rather. he concluded that the left-eye and right-eye sensations were elaborated independently, with the singleness of binocular vision being strictly psychical.

Despite the small magnitude of the differences between the various flicker conditions, the fact still remains that the differences were unequivocal among all Os. In fact, the probability of these differences, assuming that the two eyes are independent, was less than .01 by a sign test performed on the data reported in Sherrington's monograph. Nevertheless. Sherrington's conclusions were widely accepted for years as the final word regarding binocular flicker. For example. Duke-Elder (1932), in his voluminous work on ophthalmology, cites Sherrington's experiments as the paramount evidence for the independence of corresponding retinal areas.

It was not until more recently that the role of binocular integration in the perception of flicker was reexamined. This reexamination can be attributed to at least two factors. First. the fact became obvious that some of the CFFs reported by Sherrington were extremely high (e.g., 90-120 flashes/sec) in comparison to subsequent values (typically no greater than 60 flashes/sec). As pointed out by Thomas (1954), these extremely high values suggest the possibility of measurement error in Sherrington's instruments. A second factor was the subsequent literature demonstrating a number of binocular phenomena. including binocular summation of the absolute threshold, that suggest a convergence of neural activity from the two eyes.

In subsequent work on binocular flicker. the consistent result is that the CFF for binocular simultaneous presentation is higher than the CFF for binocular alternating presentation (Baker. 1952a, b.c.d: 
Baker \& Bott, 1951; Foley \& Stager, 1965: Ireland, 1950; Kinsbourne \& Coughlin, 1969; Perrin, 1954; Thomas, 1954, 1955, 1956). Moreover, the differences in CFF between these two conditions, although not of the magnitude originally anticipated by Sherrington, are larger. than those found by Sherrington (by approximately $10 \%$ ) and always statistically significant. That the difference is not limited to a particular set of stimulus conditions is evidenced by the fact that the difference was found despite variations in test field size (Baker, 1952b), test field hue (Baker, 1952b), test field intensity (Thomas, 1955), and retinal region of stimulation (Thomas, 1954). Additionally, it has been found that the presence of contours in the dichoptically viewed targets enhances the difference between binocular simultaneous and binocular alternating CFF relative to a condition in which homogeneous targets are used (Thomas, 1956).

Similarly, the difference between CFF for binocular simultaneous presentation and CFF for monocular viewing has been reported in subsequent experiments (Baker, 1952a; Crozier \& Wolf, 1941; Ireland, 1950; Thomas, 1955: Vernon, 1934) and has been found to be greater than that predicted on the basis of probability summation (Peckham \& Hart, 1960). Once again, the difference between monocular and binocular CFF invariably has been greater than the difference reported by Sherrington.

A comparatively recent development in research on flicker fusion involves the us of sinusoidally modulated light signals whose mean luminance remains constant while varying in Michelson contrast. This pattern of stimulation offers a number of advantages, including the application of powerful analytic techniques to the visual system (e.g., see Cornsweet, 1970). There appears to be only one set of experiments in which sinusoidal stimulation was used to compare monocular and binocular flicker detection performance. In several experiments, Kintz (1970) determined the contrast sensitivity for the transition between flicker and fusion over a range of temporal frequencies. For the case where the sinusoids to each eye were in phase, binocular contrast sensitivity was clearly greater than monocular for lower temporal frequencies (less than $10 \mathrm{~Hz}$ ), but for higher temporal frequencies monocular and binocular sensitivity did not differ. For the case where the sinusoids in each eye were $180 \mathrm{deg}$ out of phase, binocular sensitivity was clearly less than monocular at low frequencies, but at higher frequencies this difference was minimal. Kintz notes that the relationship between binocular and monocular performance is clearly frequency dependent and that further work is required to elucidate the mechanisms responsible for that dependence.

In view of the substantial literature demonstrating the significant role of binocular integration in the perception of flicker, it seems clear that Sherrington's original conclusion concerning the independence of corresponding retinal areas is incorrect and that, instead, there is some form of convergence of neural activity from the two eyes onto a common neural site. It is likely that Sherrington thought of summation solely in terms of the complete neural summation observed in the spinal cord and dismissed as inconsequential the small effects he obtained in vision. He correctly concluded that the integrative mechanism responsible for the singleness of binocular vision was not comparable to the summative mechanism operative in spinal reflexes. Subsequent workers, however, have considered alternative models of binocular neural summation.

One of these alternatives that deserves special consideration is the hypothesis of Crozier, for his experiments and hypothesis represent the most ambitious effort to express the quantitative relationship between monocular and binocular flicker thresholds. Crozier (Crozier \& Wolf, 1941) argued that it is necessary not only to compare monocular and binocular CFFs at all intensity values of the flickering light, but also to obtain information as to the variability of these measures, for the variability of CFF over individual trials, according to Crozier, is not due to experimental error, but instead reflects a basic property of the mechanism responsible for the flicker threshold. According to Crozier (1936), any intensity discrimination, including the detection of flicker, is the result of a statistical process. The physical stimulus has an effect on the population of excitable elements underlying the visual response, and this effect is variable even for a light of fixed intensity (I). Thus, the threshold is a measure of the dispersion or variability of the effect of $I$ on this population. One can, therefore, infer the effect of various experimental variables, including viewing condition, on this hypothetical population of excitable elements by assessing the effect of these variables on the threshold.

Applying this strategy to flicker thresholds, Crozier and Wolf obtained monocular and binocular critical intensity thresholds $\left(I_{c}\right)$ for the detection of flicker. These thresholds were obtained by gradually increasing the luminance of a light flickering at a constant rate until the $\mathbf{O}$ first reported detection of flicker. These thresholds were measured over a wide range of flicker frequencies. Binocular thresholds were always measured with in-phase flashes to the two eyes. The relationship between flicker rate and $\log I_{c}$ was best described by the normal probability integral (not to be confused with probability summation between the two eyes). That is to say, the probability of detecting flicker increased as the luminance of the flickering light increased. To evaluate the effect of binocular viewing on the hypothetical population of excitable elements underlying flicker discrimination, Crozier and Wolf compared the parameters of the monocular and binocular integral. They argued that changes in the total number of excitable elements operative would be reflected in changes in the slope and abscissa values of the normal 
integral, while changes in the potency or effectiveness of these elements would be reflected in the index of variation of $\mathrm{I}_{c}$. Results revealed that although the monocular and binocular normal integrals both reached the same asymptotic value and had the same slope. the binocular index of variation was lower than the monocular by a factor of $\sqrt{2}$. leading the authors to conclude that "for binocular flicker ... the potential effectiveness of each element is doubled, but the total number is pretty much the same [p. 530]." And it is this doubling of effectiveness of the excitable elements that increases the precision of the discrimination by the $\sqrt{2}$.

This theoretical account of binocular flicker has received support in a subsequent experiment by Thomas (1955). In this article. Thomas concludes that such an increase in the effectiveness of a fixed population of elements is plausible if it is assumed that excitation from corresponding retinal areas converge onto common cells, with excitation from both retinas producing a visual response greater than the response from stimulation of only one eye.

\section{CONTOUR RESOLUTION}

We turn now to those experiments that have examined the contribution of binocular vision to the resolution of contour information. Some of these experiments have measured binocular summation using conventional acuity measures such as Landolt rings or the Snellen chart. while others have investigated summation in the recognition of briefly presented letter forms. The consistent finding in both types of experiments is that performance is superior when two eyes are used. More recent work indicates that this superiority arises from some form of neural interaction between the two eyes.

\section{Acuity}

As early as 1920, Ferree, Rand, and Buckley (1920) observed that binocular acuity, as measured both by Snellen chart and Landolt rings, was greater than monocular acuity for nearly $90 \%$ of the individuals tested. They also found that the magnitude of the difference between monocular and binocular acuity varied widely between individuals. Similar observations were also made by Cobb (1922). However, both these early reports were primarily concerned with evaluating visual screening techniques to be used on military personnel, and consequently the theoretical implications of these results were not explored.

The first attempt to account for the superiority of binocular acuity was made by the Swedish ophthalmologist. Bárány (1946). In several clinical studies, he found that the gap in a Landolt ring could be correctly detected more often with two eyes than with one. Furthermore. in most cases. the observed binocular performance was in good agreement with predictions from the classic theorem of independence, leading Bárány to conclude that the superiority of binocular vision was due entirely to the statistical advantage of using two eyes as compared with one. This conclusion is especially interesting since it was reached without knowledge of the similar probability model proposed earlier by Pirenne (1943). (In a footnote, Bárány acknowledges that after developing this theory, he became aware of Pirenne's model.) But, of course. Bárány's conclusion in favor of probability summation, like that of Pirenne, must be questioned, since the conclusion is based on predictions from the inappropriate classic theorem of independence.

Horowitz (1949) has demonstrated that factors other than neural interaction between the two eyes can contribute substantially to the superiority of binocular acuity. Using both an Ives grating and the Landolt ring, he found that monocular acuity was best, and therefore the monocular-binocular difference was least, when 2-mm artificial pupils were used (relative to the case where normal pupils were used) and when the untested eye was exposed to a homogeneous field whose luminance was equal to the luminance of the test stimulus. Monocular acuity progressively deteriorated as the luminance difference between the tested and untested eyes increased. Horowitz speculated that this deterioration might result either from rivalry between the tested and untested eyes or from a reduction in apparent contrast due to brightness averaging. But, even under optimal conditions (i.e., artificial pupils and equal luminance to both eyes), binocular acuity was still superior to monocular acuity. This superiority has more recently been confirmed in a carefully performed experiment by Kahneman, Norman, and Kubovy (1967).

In the experiments mentioned so far, acuity has been measured using high-contrast test objects and has been expressed in terms of either minimal separable distance or probability of seeing. In all these experiments, the superiority of binocular acuity has been modest. There is one study reported in which a fundamentally different technique was used to measure acuity, and the resulting difference between monocular and binocular acuity was large. Campbell and Green (1965) measured monocular and binocular contrast thresholds for detection of grating patterns whose luminance profiles were modulated sinusoidally about a constant mean luminance. Over a wide range of spatial frequencies. the binocular threshold was consistently lower than the monocular. The average ratio of binocular to monocular contrast sensitivity was not significantly different from $\sqrt{2}$, indicating an improvement for binocular viewing on the order of $42 \%$. Campbell and Green argued that this $\sqrt{2}$ improvement could be explained by assuming that signals from the two eyes are summed and that the signals contain noise that is uncorrelated between the two eyes. They poin out that since the standard error of the sum of $n$ independent measures of a noisy process is 
reduced by $\sqrt{n}$, the use of two eyes would permit a $\sqrt{2}$ lower contrast to be detected. The authors emphasize that physiological summation between the eyes is a necessary assumption of this signal-to-noise model.

This model is reminiscent of Crozier's theory, which was described in the section on flicker thresholds. Both models attribute the increase in sensitivity that obtains with binocular viewing to a decrease in the noise, or variability, associated with the binocular response. Indeed, a model of this form may provide the most parsimonious account of the rather ubiquitous $\sqrt{2}$ improvement factor that has been obtained for the increment threshold, flicker sensitivity, and spatial acuity.

\section{Form Recognition}

The role of binocular summation in the recognition of briefly presented forms has recently been investigated by Eriksen and his colleagues (Eriksen, Greenspon, Lappin, \& Carlson, 1966; Eriksen \& Greenspon, 1968). In these experiments, a three-alternative, forced-choice procedure was used to measure Ss' accuracy in identifying a briefly exposed letter presented either monocularly or binocularly. The consistent finding was that form recognition accuracy was significantly higher with two eyes than with one. When letters were presented within $50 \mathrm{msec}$ of each other and to corresponding retinal areas, binocular performance was in excess of that predicted by a two-state model of independence; for intervals greater than $50 \mathrm{msec}$ or for stimulation of noncorresponding areas, binocular performance was equivalent to the prediction of the two-state model. Finally, in both experiments, it was found that when the energy to a single eye was doubled, the recognition accuracy was significantly higher than that obtained with binocular stimulation, a result which led Eriksen to conclude that binocular summation, although greater than probability, was not complete.

While the work of Eriksen clearly demonstrates binocular summation in the recognition of briefly presented letters, a recent experiment by Townsend (1968) raises the possibility that this binocular advantage may be limited to the case where forms are presented within a homogeneous field. In Townsend's experiment, Ss were required to report which of 2 target letters appeared within a briefly presented display composed of 16 letters arranged in a foveally viewed 4 by 4 matrix. The target letter was randomly located in one cell of the matrix and the remaining cells were filled with nontarget letters. Townsend found that the binocular hit rate was best described by the average of the monocular hit rates, indicating that binocular vision added nothing to the accuracy of letter recognition. A similar pattern of results was obtained in several unpublished experiments performed by us (i.e., the writers in collaboration with Curtis McIntyre). Specifically, we measured forced-choice recognition thresholds for target letters presented either alone or embedded in an array of nontarget letters. For a single letter, summation was in excess of probability, replicating the findings of Eriksen; when the target letter was surrounded by 7 or 13 nontarget letters, binocular performance was no better than monocular, even though for both conditions hit rates were above chance, replicating the results of Townsend.

The failure to find evidence for summation with complex displays is surprising in light of the considerable evidence that binocular vision facilitates performance on a number of psychophysical tasks. One might argue that recognition of letters surrounded by other letters is so difficult a task that summation between the eyes was not sufficient to improve performance. But even if this were true, it is not obvious why one does not observe at least some improvement in binocular performance simply from probability summation. Certainly the logic underlying the probability-summation hypothesis is not limited to simple threshold tasks. Interestingly, a somewhat similar finding has been reported by Casperson and Schlosberg (1950), who measured the detection of dot numerosity during monocular and binocular viewing. They found that when the number of dots did not exceed four, the results could be described by a binocular probability summation model, but that for more than four dots, binocular performance declined to the monocular level.

Although the evidence is meager, one could entertain the possibility that the superiority of binocular performance, whether the result of neural or probability summation, is limited to rather simple stimulus displays with relatively few contours. In this regard, recall that Fry and Bartley (1933) hypothesized the operation of two processes within the binocular visual system, a summation process and an inhibition process, and that the final binocular response is a function of some combination of the two. Consistent with this hypothesis are those experiments demonstrating interocular inhibitory effects (e.g., Flom, Heath, \& Takahashi, 1963). On the basis of this dual process model, one could speculate that, with complex displays containing numerous contours, the contribution of inhibition is greater than that of summation, thus eliminating any superiority of binocular viewing. In any case, the apparent absence of summation with complex displays is intriguing and suggests the need for a more systematic investigation into the boundary conditions surrounding the operation of binocular summation.

\section{GENERAL DISCUSSION}

It seems clear that, for all visual tasks so far examined, binocular performance is superior to monocular. For such threshold tasks as increment detection, form recognition, acuity, and flicker fusion, binocular performance is greater than that predicted by probability summation models, thereby justifying the 
conclusion that neural mechanisms are responsible for summation. With respect to suprathreshold tasks, such as brightness matching and reaction time. the interpretation of the results is not so clear-cut. The research on brightness is besieged by questions concerning the appropriate monocular baseline for deciding whether or not an enhancement of brightness occurs under the binocular condition. The research on reaction time has not yet distinguished between enhanced performance attributable to probability summation and that due to neural summation.

In experiments that have revealed neural summation, the absolute magnitude of summation has not been large, and summation has been observed for a rather restricted range of stimulus conditions. For instance. in almost all the threshold experiments, i.e., those summation experiments employing threshold tasks. the stimuli are simple configurations presented against homogeneous backgrounds, the stimuli to each eye are identical in terms of all stimulus energy parameters, and the stimuli are carefully aligned either to fall on corresponding portions of each retina or to fall on clearly separate noninteractive portions of each retina. In the majority of experiments, the stimuli are presented simultaneously to each eye during the binocular condition,

It is not known if this set of conditions is essential for producing summation or whether other conditions would reduce or enhance the magnitude of summation. It is possible that summation occurs only when the eyes receive identical packets of stimulus energy. and for cases where the packets are not identical the summation mode of operation switches to the inhibitory mode. One can imagine an increment detection experiment where the stimulus is vertically oriented in one eye while an equally detectable horizontally oriented stimulus is presented to the other eye. Although the energy delivered to the central locus would be the same as if both stimuli had comparable orientations, it may be that the difference in orientation of the stimuli would give rise to an inhibitory response rather than a summative one. Or. suppose a circular increment flash to one eye was made detectable by virtue of its being large and dim while its equally detectable partner stimulus was relatively small and bright. Again, although the energy delivered to the central locus would be the same, it is possible that the difference in the area and intensity coding of the stimuli would preclude summation. One pervading theoretical question raised by these speculations concerns the extent to which the binocular system at threshold operates as a general integrator of stimulus energy, or whether such integration is confined to the case where the monocular stimuli are perfectly equivalent. Experiments that systematically vary stimulus characteristics between the eyes may reveal the way in which the binocular visual system initially: classifies the similarity of stimuli. These data may be helpful in understanding the suprathreshold situation where differences in stimulation (except those yielding stereopsis) eventually provoke the inhibitory action of binocular rivalry.

With respect to conditions that may enhance summation, differences in stimulation vis-à-vis corresponding regions of each eve due to nonconjugate microsaccadic eye movements could serve to reduce summation. An experiment that eliminated these eye movements by means of an image-stabilization procedure may reveal an increase in summation. The practice of aligning the monocular stimuli so that they fall on corresponding parts of each retina insures that the stimuli will appear fused, but at the same time minimizes the likelihood that the stimuli will be seen in the apparent depth produced by stereopsis. Perhaps if the stimuli were aligned so as to be sufficiently disparate to generate stereoscopic depth, the stereopsis mechanism would interact in a facilitatory way with the summation process, thereby yielding a larger summation effect. Although the practice of presenting both monocular stimuli simultaneously to the eyes during the binocular condition would seem to maximize the opportunity for energy integration, it is possible that integration would be facilitated if the stimuli were presented successively. Indeed, in the experiments by Matin (1962) and by Eriksen and Greenspon (1968), the data suggest that performance may be enhanced when the stimuli are separated by a brief temporal interval.

The purpose of this speculative excursion into the domain of hypothetical experiments is to illustrate that it is not unreasonable to suppose that various stimulus conditions might yield varying amounts of summation or even inhibition. Until these experiments are performed or rendered untenable on logical grounds, the conclusion that the summative effects observed so far represent the general operating characteristics of the binocular visual system seems premature. We are suggesting that there may be no single mode of summation applicable for all functions of the visual system, but rather, variations that are contingent upon the conditions of stimulation. Although this may appear to be an unparsimonious position, the phenomena of binocular fusion, binocular rivalry, and stereopsis demonstrate that radically different operating characteristics of the visual system can be manifest by appropriate stimulus conditions.

\section{The Relationship of Neurophysiological Data to Psychophysical Research on Binocular Summation}

The investigations of single neuron activity in the visual cortex of monkeys (Hubel \& Wiesel, 1968) and cats (e.g., Hubel \& Wiesel, 1962) demonstrate considerable interocular interaction within the visual cortex. Moreover, recent evidence for such interaction in the lateral geniculate of cat has been reported as well (e.g. see review by Rodieck. 1971). The question naturally arises as to what implications single-unit data have for the problem of summation as it is assessed in 
the psychophysical experiment. Since it has been demonstrated that neural summation occurs at the level of the single cell when a stimulus is presented to each eye so that it falls within the same receptive field of that cell, it might be argued that the question of neural summation has already been answered. Yet it is not clear how this summative response of the single cell is related to the overall function of the visual system as it is revealed by the psychophysical experiment. The increased firing rate of cells in the visual cortex still represents an early stage in the visual system, and an increase in firing rate is not readily related to the final output of the system. Surely, the assumption that the output of the system directly reflects the increase in firing rate is difficult to justify, since that assumption leads to considerably more behavioral summation than has ever been observed. For example, the cells observed by Burns and Pritchard (1968) exceed the resting level by $200 \%$. The problem of developing an adequate theory that relates the rate of firing of nerve cells to behavioral responses is a very general one, not confined to the specific issue of binocular summation. Until progress is made toward a solution to that general problem, it would seem that the recent data on single cells does not contribute materially to our understanding of binocular summation. It was the anatomical evidence for convergence of the monocular pathways that sponsored an initial interest in behavioral experiments in binocular summation; the single-cell data serves to confirm and extend the anatomical analysis.

These comments are not meant to indicate that the physiological data have no relevance for the problem of binocular summation, but simply to indicate that at the present time the data are inadequate to solve the issues raised by the behavioral experiments. In the future, we expect that the physiological research in combination with the psychophysical line of attack will serve to develop a much more comprehensive and satisfactory theory of binocular interaction.

\section{The Functional Significance of Binocular Summation}

Even though we have suggested some stimulus conditions that might reveal enhanced summation, the fact remains that the available data reveal only a modest contribution by: binocular vision to visual performance. And, as this review indicates, the demonstration of that contribution has not been easy. Only the more recent experiments consistently have been able to demonstrate a superiority in binocular performance relative to monocular. These comments provide a context for answering the question raised in the introduction, namely, are two eyes better than one? The answer to that question seems to be, yes, two eyes are better than a single eye for many visual tasks, but not by very much. That answer must be further qualified by the fact that the superior binocular performance has been observed only for situations where a target of simple configuration is seen against a homogeneous background. In those experiments that have employed more complex displays, approximating more closely the viewing conditions of a natural environment, no evidence for binocular superiority has been observed.

The marginal contribution made by a second eye raises the question as to the adaptive advantage of binocular vision, an advantage that must be sufficiently strong to maintain the complicated mechanism necessary for the coordinated operation of two eyes. One answer is that the eyes are arranged binocularly in order to extract depth information from the environment through the mechanism of stereopsis. On this view, the extensive convergence between the monocular inputs evolved in order for the development of a stereoptical mechanism and not for any significant increase in visual efficiency. Indeed, it has been suggested that the principal function of convergence is the control of the movements of the eyes so that they remain coordinated in order to permit the occurrence of patent stereopsis. This suggestion has been made by Sherrington (1906) and more recently by Garth Thomas (1955) and by Burns and Pritchard (1968). If this is the correct interpretation, then the small amount of improvement in visual efficiency found during binocular vision may be regarded as essentially an epiphenomenon of the more fundamental task of controlling the direction of gaze.

\section{Final Comment}

This review has raised more questions than it has answered. Nevertheless, it is clear that progress has been made on the question of binocular summation as it was originally posed at the beginning of this century. It can now be concluded that binocular summation in excess of probability summation can be reliably demonstrated for a variety of visual tasks. And the work leading to this conclusion has been accompanied by improvements in methodology and analytical procedures that will prove useful in future work. Although this amount of progress may appear small, it should be remembered that during this century considerable progress has been made in understanding the operation of the monocular visual pathways. Given our firmer understanding of the monocular situation, it is likely that an intensive attack can soon begin upon the next stage in the chain, the binocular stage, where the monocular systems interact. We are optimistic, then, that many of the questions raised in this review will receive answers in a relatively short period of time.

\section{APPENDIX A}

To use the two-state model to predict probability summation, it is necessary to correct the monocular hit rates, $P_{L}$ and $P_{R}$, for guessing. As Eriksen and 
Greenspon (1968) have shown. this correction is provided by

$$
G_{L}=(1-1 n)^{-1}\left(1-P_{L}\right)
$$

and

$$
G_{R}=(1-1 / n)^{-1}\left(1-P_{R}\right)
$$

where $G_{L}$ and $G_{R}$ are the total percentage of guess trials (both correct and incorrect) for the left and right eyes. respectively, and $1 \mathrm{n}$ is the a priori probability of a correct guess. Thus, for binocular viewing. $1-G_{L} \cdot G_{R}$ provides an estimate of the proportion of trials when the $\mathrm{S}$ correctly perceived with either one eye or the other. or both. To obtain the final binocular prediction. it is necessary to add to this value the proportion of trials on which the $\mathrm{S}$ failed to perceive with either eye but did guess correctly. which is given by $1 / n\left(G_{L} \cdot G_{R}\right)$. Thus. for the binoculat situation. the performance predicted on the basis of probability summation, assuming the high-threshold two-state model. is given by

$$
1-G_{R} \cdot G_{L}+1 / n\left(G_{R} \cdot G_{L}\right) \text {. }
$$

\section{REFERENCES}

Abney, W. de W.. \& Watson. W. The threshold of vision for different colored lights. Philosophical Transactions of the Royal Society. A. 1916. 216.91-128.

Baker. C. H. The dependence of binocular fusion on timing of peripheral stimuli and on central process. I. Symmetrical flicker. Canadian Journal of Psychology. 1952a, 6. 1-10.

Baker. C. H. The dependence of binocular fusion on timing of peripheral stimuli and on central process. I. Symmetrical flicker (continued). Canadian Journal of Psychology 1952b. 6. 84.91.

Baker. C. H. The dependence of binocular fusion on timing of peripheral stimuli and on central process. II. Asymmetrical flicker. Canadian Journal of Psychology, 1952c. 6. 123-130.

Baker. C. H. The dependence of binocular fusion on timing of peripheral stimuli and on central process. III. Cortical flicker. Canadian Journal of Psychology. 1952d. 6. 151-163.

Baker. C. H.. \& Bott. E. A. Studies on visual flicker and fusion. II. Effects of timing of visual stimuli on binocular fusion and flicker. Canadian Journal of Psychology, 1951. 5. 9-17.

Bárány. F. A theory of binocular visual acuity and an analysis of the variability of visual acuity. Acta Ophthalmologica. 1946. 24, 63-92.

Bartlett, N. R. Thresholds as dependent on some energy relation and characteristics of the subject. In C. Graham (Ed.), Vision and visual perception. New York: Wiley. 1965.

Bartlett. N. R. \& Gagné. R. M. On binocular summation at threshold. Journal of Experimental Psychology. 1939. 25. 91-99.

Bartley, S. H. Some parallels between pupillary reflexes and brightness discrimination. Journal of Experimental Psychology: 1943.32. 110-122

Battersby, W. S.. \& Defabaugh. G. L. Neural limitations of visual excitability: After-effects of subliminal stimulation. Vision Research. 1969. 9.757-768.

Braddick. O. Binocular interaction and signal detection theory. Vision Research. 1972. 12. 1435-1437.

Burns. B. D.. \& Pritchard. R. Cortical conditions for fused binocular vision. Journal of Physiology. 1968. 197. 149-171.

Campbell. F. W.. \& Green. D. G. Monocular versus binocular visual acuity Vature. 1965. 208. 191-192.
Casperson. R. C.. \& Schlosberg. H. Monocular and binocular intensity thresnolds for fields containing 1-7 dots. Joumat of Experimental Psychology. 1950.40.81-92.

Cobb. P. W. Individ: al variations in retinal sensitivity and their correlation with ophthalmological findings. Journal of Experimental Psyclology, 1922. 5. 227-246.

Collier. G. Probability of response and intertrial association as functions of monocular and binocular stimulation. Journal of Experimental Psychology. 1954. 47. 75-83.

Collier. G.. \& Kubzansky. P. The magnitude of binocular summation as a function of the method of stimulus presentation. Journal of Experimental Psychology, 1958. 56. 355-361.

Cook. T. W. Binocular and monocular relations in foreal dark adaptation. Psychological Monographs. 1934. 45 Whole o. 202).

Cornsweet. T. N. Iisual perception. New York: Academic Press. 1970.

Crozier. $W, J$. On the sensory discrimination of intensities. Proceedings of the National Academy of Science. 1936. 22. $412-416$.

Crozier. W. J.. \& Holway, A. H. Theory and measurement of visual mechanisms. I. A visual discriminometer. I1. Threshold stimulus intensity and retinal position. Joumal of General Physiology. 1939a. 22. 341-364.

Crozier. W. J.. \& Holway. A. H. Theory and measurement of visual mechani ms: III. $\Delta \mathrm{I}$ as a function of area. intensity. and wavelength for monocular and binocular stimulation. Journal of General Phy siology. 1939b. 23. 101-141.

Crozier. W. J.. \& Wolf. E. Theory and measurement of visual mechanisms: IV. Critical intensities for visual flicker. monocular and binocular. Journal of General Physiology. 1941. 24, $505-534$

Dawson. S. Binocular and uniocular discrimination of brightness. British Journal of Psychology. 1913, 6, 78-108.

DeSilva. H. R., \& Bartley. S. H. Summation and subtraction of brightness in binocular perception. British Journal of Psychology. 1930. 20, 242-252.

Doherty. M. E.. \& Keeley. S. M. A Bayesian prediction of four-look recognition performance from one-look data. Perception \& Psychophysics. 1969, 5, 362-364.

Doherty, M. E., \& Keeley. S. M. On the identification of repeatedly presented, brief visual stimuli. Psychological Bulletin. 1972.78.142-154.

Downey. J. Determination of minimum light sense and retinal dark adaptation. American Journal of Ophthalmology. 1919. 2. 13-20.

Duke-Elder. W. W. Textbook of ophthalmolog: Vol. 1. London: Kimpton. 1932.

Engel. G. R. The visual process underlying binocular brightness summation. Vision Research. 1967.7.753-767.

Engel. G. R. The autocorrelation function and binocular brightness mixing. Vision Research, 1969, 9.1111-1130.

Engel. G. R. Tests of a model of binocular brightness. Canadian Journal of Psychology. 1970. 24. 335-352.

Eriksen. C. $W$. Independence of successive inputs and uncorrelated error in visual form perception. Journal of Experimental Psychology. 1966. 72. 26-35.

Eriksen. C. W.. \& Greenspon. T. S. Binocular summation over time in the perception of form at brief durations. Journal of Experimental Psychology, 1968. 76. 331-336.

Eriksen. C. W.. Greenspon. T. S.. Lappin. J. S. . \& Carlson. W. A Binocular summation in the perception of form at brief durations. Perception \& Psychophysics. 1966. 1. 415-419.

Eriksen. C. W.. \& Lappin. J. S. Internal perceptual system noise and redundancy in simultaneous inputs in form identification. Psychonomic Science. 1965. 2.351-352.

Eriksen. C. W.. \& Lappin. J. S. Independence in the perception of simultaneously presented forms at brief durations. Journal of Experimental Prichology, 1967.73. 468.472. 
Fechner. G. T. Elemente der Psychophy'sik. Leipzig: Breitkopf \& Härtel. 1860.

Feller. W. An introduction to probability theory and its applications. New York: Wiley, 1960.

Ferree, C. W.. Rand, G., \& Buckley, D. Study of ocular functions with special reference to the lookout and signal service of the navy. Journal of Experimental Psychology, 1920, 3, 347-356.

Flom, M. C., Heath, G. G., \& Takahashi, E. Contour interaction and visual resolution: Contralateral effects. Science, 1963, $142,979-980$.

Foley, P. J.. \& Stager, P. The phase difference function in binocular flicker. Canadian Journal of Psychology, 1965, 19, 47-55.

Forbes, L. M., \& Mote, F. A. A comparison of the variability of binocular and monocular threshold measurements during dark adaptation in the human eye. Journal of Comparative \& Physiological Psychology, 1956, 49, 431-436.

Fry, G. A. The relation of accommodation to the suppression of vision in one eye. American Journal of Ophthalmology, 1936, $19,135-138$

Fry, G. A., \& Bartley, S. H. The brilliance of an object seen binocularly. American Journal of Ophthalmology, 1933, 16, $687-693$

Garner, W. R., \& Aforton, J. Perceptual independence: Definitions, models, and experimental paradigms. Psychological Bulletin, 1969, 72, 233-259.

Graham, C. H. An investigation of binocular summation: I. The fovea. Journal of General Psychology, 190, 3, 494-509.

Graham, C. H. An investigation of binocular summation: II. The periphery. Journal of General Psychology, 191, 5, 311-327.

Graham, C. H. Neural correlates. In C. Murchison (Ed.), $A$ handbook of general experimental psychology. Worcester: Clark University Press, 1934. Pp. 829-879.

Graham, C.. Brown, R. H., \& Mote, F. A. The relative size of stimulus and intensity in the human eye. Journal of Experimental Psychology, 1939, 24, 555-573.

Granit, R. Charles Scott Sherrington: $A$ biography of the neurophy siologist. New York: Doubleday, 1967.

Green, D. M., \& Swets, J. A. Signal detection theory and psychophysics. New York: Wiley, 1966.

Guth, S. L. On probability summation. Vision Research, 1971, $11,747-750$.

Hecht, S. The nature of foveal dark adaptation. Journal of General Physiology, 1921, 4, 113-139.

Horowitz, M. W. An analysis of the superiority of binocular over monocular visual acuity. Journal of Experimental Psychology, $1949,39,581-596$.

Hubel, D. H., \& Wiesel, T. N. Receptive fields, binocular interaction and functional architecture in the cat's visual cortex. Journal of Physiology, 1962, 160, 106-154.

Hubel, D. H., \& Wiesel, T. N. Receptive fields and functional architecture of monkey striate cortex. Journal of Physiology, 1968, 195, 215-243.

Ireland, F. H. Comparison of critical flicker frequencies under conditions of monocular and binocular stimulation. Journal of Experimental Psychology, 1950, 40, 282-286.

Kahneman, D., Norman, J., \& Kubovy, M. Critical duration for the resolution of form: Centrally or peripherally determined? Journal of Experimental Psychology, 1967, 73, 323-327

Keeley, S. M., \& Doherty, M. E. Simultaneous and successive presentations of single-featured and multi-featured visual forms: Implications for the parallel processing hypothesis. Perception \& Psychophysics, 1968, 4, 296-298.

Keeley, S. M., \& Doherty, M. E. Bayesian aggregation of independent successive visual inputs. Journal of Experimental Psychology, 1971a, 90, 300-305

Keeley, S. M., \& Doherty, M. E. A Bayesian prediction of multiple look identification performance from one-look data: The effect of unequal prior probabilities. Perception \& Psychophysics, 1971b, 10, 119-122.
Kincaid, W. M., Blackwell, H. R., \& Kristofferson, A. B. Neural formulation of the effects of target size and shape upon visual detection. Journal of the Optical Society of America, 1960, $50,143-148$.

Kinsbourne, M., \& Coughlin, P. J. The demonstration of dichoptic flicker effects by the use of Polaroid. Quarterly Journal of Experimental Psychology, 1969, 21, 67-68.

Kintz, R. T. A comparison of monocular and binocular temporal resolution in human vision. Unpublished doctoral dissertation, University of Rochester, 1970.

Laird, D. Studies relating to the problem of binocular summation. Journal of Experimental Psychology, 1924, 7. 276-290.

LeGrand, Y. Form and space vision. Bloomington: Indiana University Press, 1967.

Leibowitz, H. \& Walker, L. Effect of field size and luminance on the binocular summation of suprathreshold stimuli. Journal of the Optical Society of America, 1956, 46, 171-172.

Levelt, W. J. M. On binocular rivalry. (Dissertation, Leiden University, 1965.) (2nd ed.) The Hague: Mouton, 1968.

Lythgoe, R. J., \& Phillips, L. R. Binocular summation during dark adaptation. Journal of Physiology, 1938, 91, 427-436.

Matin, L. Binocular summation at the absolute threshold for peripheral vision. Journal of the Optical Society of America, $1962,52,1276-1286$

McGill, W. J. Stochastic latency mechanisms. In R. D. Luce, R. R. Bush, and E. Galanter (Eds.), Handbook of mathematical psychology. Vol. 1. New York: Wiley, 1963. Pp. 309-360.

Minucci, P. K., \& Connors, M. M. Reaction time under three viewing conditions: Binocular, dominant eve. and non-dominant eye. Journal of Experimental Psychology, $1967,67,268-275$.

Peckham, R. H. \& Hart, W. M. Binocular summation of subliminal repetitive visual stimulation. American Journal of Ophthalmology, 1960, 49, 1121-1125.

Perrin, F. H. A study of binocular flicker. Journal of the Optical Society of America, 1954, 44, 60-69.

Piper, $H$. Über das Helligkeilsverhaltnis monokular und binokular a usgeloster Lichtempfindungen. Zeitschrift für Sinnesphysiologie, 1903, 32, 161-176. [Cited by T.W. Cook, Binocular and monocular relations in foveal dark adaptation. Psychological Monographs, 1934, 45 (Whole No. 202).]

Pirenne, M. H. Binocular and uniocular thresholds in vision. Nature, 1943, 152, 698-699.

Pirenne, M. H., \& Marriott, F. H. C. The quantum theory of light and the psychophysiology of vision. In S. Koch (Ed.) Psychology: A study of a science. Study I: Conceptual and systematic. Vol. 1. Sensory, perceptual, and physiological formulations. New York: McGraw-Hill, 1959. Pp. 288-361.

Poffenberger, A. T. Reaction time to retinal stimulation with special reference to the time lost in conduction through nerve centers. Archives of Psychology, 1912, 23, 1-73.

Polyak, S. L. The vertebrate visual system. Chicago: University of Chicago Press, 1957

Reeves, P. Rate of pupillary dilation and contraction. Psychological Review, 1918, 25, 330-340.

Rodieck, R. W. Central nervous systems: Afferent mechanisms. Annual Review of Physiology, 1971, 33, 203-240.

Roelofs, C., \& Zeeman, W. Zur Frage der binokularen Schwellenwerte. v. Graefe's Archiv für Ophthalmologie, 1914, $88,1-27$.

Shaad, D. Binocular summation in scotopic vision. Journal of Experimental Psychology, 1935, 4, 391-413.

Sherrington, C. S. On binocular flicker and the correlation of activity of "corresponding retinal points." British Journal of Psychology, 1904, 1. 26-60.

Sherrington, C. S. The integrative action of the nervous system. New Haven: Yale University Press, 1906. Republished 1947, 1961

Stevens, J. C. Brightness function: Binocular versus monocular 
stimulation. Perception \& Psychophysics. 1967, 2, 451-454.

Swets. J. A. Is there a sensory threshold? Science, 1961, 134, 168-177.

Swets, J. A. (Ed.), Signal detection and recognition by human observers: Contemporary readings. New York: Wiley, 1964.

Teller. D. Y.. \& Galanter, E. Brightness, luminances, and lechner's paradox. Perception \& Psychophysics, 1967, 2, 297-300.

Thomas, G. J. The effect on critical flicker frequency of interocular differences in intensity and phase relations of flashes of light. American Journal of Psychology, 1954, 67, 632-646.

Thomas. G. J. A comparison of uniocular and binocular critical flicker frequencies: Simultaneous and alternate flashes. A merican Journal of Psychology, 1955, 68, 37-53.

Thomas, G. J. Effect of contours on binocular CFF obtained with synchronous and alternate flashes. American Journal of Psychology, 1956, 69, 369-377.

Thomson. L. C. Binocular summation within the nervous pathways of the pupillary light reflex. Journal of Physiology. 1947, 106. 59-65.

Townsend, J. T. Binocular information summation and the serial processing model. Perception \& Psychophysics. 1968, 4. 125-128.

Vernon. M. D. The binocular perception of flicker. British Journal of Psychology, 1934, 24, 251-274.

Wald, $G$. Area and visual threshold. Journal of General Physiology, 1938, 21, 269-287.

Walls, G. L. A theory of ocular dominance. Archives of Ophthalmology, 1951, 45. 387-412.

Westendorf, D. H., Blake, R. R., \& Fox, R. Binocular summation of equal-energy flashes of unequai duration. Perception \& Psychophysics, 1972.12. 445-448.

Whittle, P.. \& Challands, P. D. C. The effect of background luminance on the brightness of flashes. Vision Research, 1969, 9. 1095-1110.

Wolf, E., \& Zigler. M. J. Uniocular and binocular scotopic responsiveness of the peripheral retina. Journal of the Optical Society of America, 1959, 49, 394-398.

Wolf. E.. \& Zigier, M. J. Effects of uniocular and binocular excitation of the peripheral retina with test fields of variou shapes on binocular summation. Journal of the (optical Society of America. 1963. 53.1199-1205.

Wolf, E., \& Zigler, M. J. Excitation of the peripheral retina with coincident and disparate test fields. Journal of the Optical Society of America. 1965, 55, 1517-1519.

Woodworth, R. S., \& Schlosberg, H. Experimental psychology. New York: Holt, Rinehart, \& Winston, 1954.

Zigler, M. J., \& Wolf, E. Scotopic parafoveal sensitivity. American Journal of Psychology, 1958, 71, 186-198.

\section{NOTES}

1. Although we have tried to make the review as complete as possible, it is very likely that we have omitted relevant material. particularly items residing in less publicized places. To correct our omissions, we solicit information about these items so that we may prepare a supplemental bibliography for publication in this journal.

2. The term "absolute threshold" will be used in this paper to refer to thresholds for the detection of a spot of light presented against a dark background. Use of the term carries no assumptions about the stimulus conditions (e.g., degree of adaptation, retinal locus of stimulation); rather, these conditions will be described for each experiment.

3. An interesting analog of Fechner's paradox has been described by Bartley (1943). He observed that when the two eyes were exposed to unequal light intensities, the degree of pupillary constriction was intermediate between that obtained when either eye alone was stimulated.

4. The notion of binocular brightness averaging did not. of course, originate with Levelt. Recall that Sherrington (1906, p. 373) proposed a similar model. There is good reason to suspect (Granit, 1967) that Sherrington was influenced by Hering, who utilized the concept of brightness averaging within his general theory of vision.

\footnotetext{
(Received for publication May 22, 1972; revision received Mąrch 26, 1973.)
} 